This item was submitted to Loughborough's Research Repository by the author.

Items in Figshare are protected by copyright, with all rights reserved, unless otherwise indicated.

\title{
The circular city with heterogeneous firms
}

PLEASE CITE THE PUBLISHED VERSION

PUBLISHER

(c) Loughborough University

VERSION

VoR (Version of Record)

LICENCE

CC BY-NC-ND 4.0

REPOSITORY RECORD

Alderighi, Marco, and Claudio Piga. 2019. "The Circular City with Heterogeneous Firms". figshare. https://hdl.handle.net/2134/4254. 
This item was submitted to Loughborough's Institutional Repository (https://dspace.lboro.ac.uk/) by the author and is made available under the following Creative Commons Licence conditions.

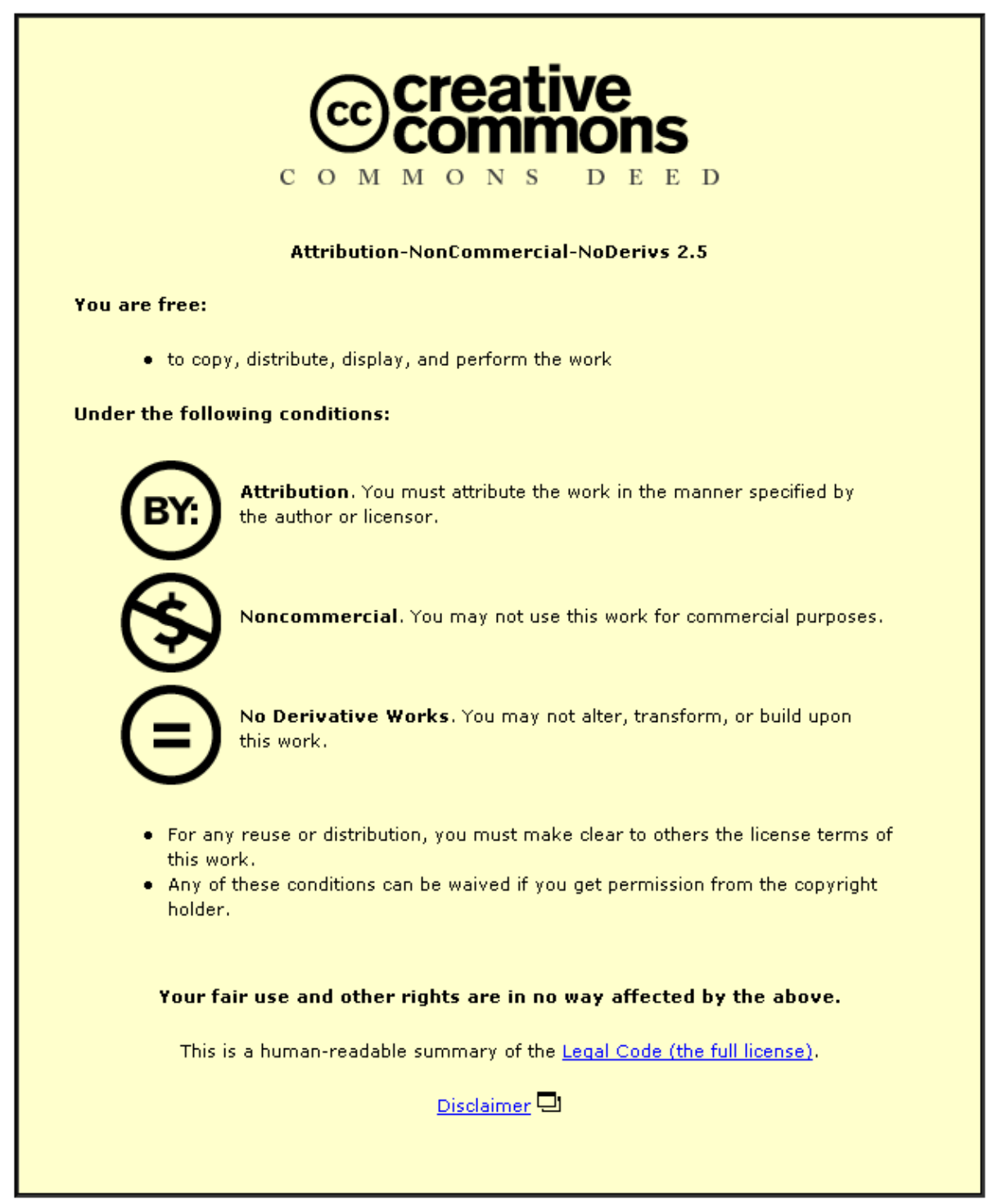

For the full text of this licence, please go to: http://creativecommons.org/licenses/by-nc-nd/2.5/ 
ISSN 1750-4171

\section{DEPARTMENT OF ECONOMICS}

\section{DISCUSSION PAPER SERIES}

\section{The Circular City with Heterogeneous Firms}

Marco Alderighi
Claudio A. Piga

\section{WP 2008 - 03}

Department of Economics Loughborough University Loughborough

LE11 3TU United Kingdom

Tel: + 44 (0) 1509222701

Fax: + 44 (0) 1509223910

http://www.lboro.ac.uk/departments/ec 


\title{
The Circular City with Heterogeneous Firms
}

\author{
Marco Alderighi ${ }^{\mathrm{a}}$ \\ Università della Valle d'Aosta, Italy. \\ Università Bocconi, Milano, Italy.
}

\author{
Claudio A. Pigab \\ Loughborough University, Loughborough, UK. \\ RCEA, Rimini, Italy.
}

July 22, 2008

\begin{abstract}
The paper extends the Salop model of localized competition by allowing firms to have heterogeneous costs. We provide a general but highly tractable analytical solution for the equilibrium prices, and we study the long-run properties of the model using two different entry games. We show that cost heterogeneity affects the efficiency of the market equilibrium by increasing welfare and inducing less excessive entry. Further, we illustrate the positive effects of the existence of a selection mechanism, which induces less efficient firms not to start production. The model also replicates some recent results on dense markets.
\end{abstract}

JEL classification: L11, D61.

Keywords: Localized competition; market efficiency, cost heterogeneity; large markets.

\section{Introduction}

The economic analysis of monopolistically competitive market structures has developed around two main methodological workhorses: the Hotelling-Salop and the Dixit-StiglitzKrugman (D-S-K) approaches. In the former, the market is represented by a circle where all firms sell an identical product whose differentiation is a direct function of the distance between the locations, defined in either a geographical or a product characteristics' space, of a consumer and of the firms serving the market. The latter is based on the idea that consumers have a preference for varieties so that each firm produces a different product. Which of these set-ups is used to analyze a particular market is often an empirical matter that crucially depends on whether competition is localized - which would favor the use of the Hotelling-Salop approach - or global.

Chamberlin (1956)'s original view of monopolistic competition encompassed the notions that firms are different and that product differentiation among firms imply differences

\footnotetext{
${ }^{a}$ E-mail: m.alderighi@univda.it.

${ }^{\mathrm{b}}$ Corresponding Author. E-mail: c.a.g.piga@lboro.ac.uk
} 
in firms characteristics, e.g. managerial abilities may differ, access to input markets may vary, etc. Nonetheless, the two main theoretical approaches to monopolistic competition have generally assumed identical firms. The need to better link empirical results with the theoretical literature calls for the development of models where firms' heterogeneity is explicitly taken into account. For example, recently Melitz and Ottaviano (2008) have, amongst other things, extended the D-S-K model by allowing different marginal costs of production.

While the implications of introducing cost heterogeneity in the Hotelling linear city model have been amply investigated in the literature (e.g., see: Bester, 1992; Piga, 1998), the Salop (1979)'s circular city model has been generally studied by assuming firms with identical marginal costs, with the notable exceptions of Syverson (2004) and Vogel (2008). In this paper we delve deep into the short- and long-run properties of the Salop's model when firms' costs are heterogeneous, thereby clarifying and extending some previous results obtained in the existence literature on localized competition.

We begin by offering a more in-depth understanding of firms' price setting behaviour in the Salop's model, by allowing any generic number of firms to differ in their efficiency levels. Assuming that firms' costs and locations are common knowledge, our analytical solution of the equilibrium prices generalizes and confirms the intuitions put forward in Syverson (2001) that prices should be a weighted average of all the firms's costs, that weights should decrease with distance and should sum to one. Weights are also found to be independent of the cost differential between any pairs of firms and turn out to converge very quickly to a stable value as the number of firms increases. Such properties of the equilibrium weights are not imposed on the solution but constitute its intrinsic characteristics. Finally, allowing for cost heterogeneity entails that a highly efficient firm may have an incentive to drive a highly inefficient neighbour from the market; to prevent this from occurring we derive the condition on the maximum allowable cost gap such that the "no mill price undercutting" rule, first proposed by Eaton and Lipsey (1978), holds in a subgame perfect Nash-price equilibrium.

After deriving a general result for the full information equilibrium price, the paper investigates how cost heterogeneity affects the long-run equilibrium market structure in two different entry games, each having price competition as the final stage. Contrary to the two-stage perfect information game in Salop (1979), the decision to enter is taken under uncertainty. Indeed, the first entry game here assumes firms learn both their and their rivals' production costs and locations only after incurring a sunk entry fee. Because expected profit increases with the cost variance, an interesting result is that under a freeentry zero profit condition more firms choose to operate than in the case where firms have 
all identical costs. Spulber (1995) shows a similar relationship between expected profits and cost uncertainty for the case of Bertrand competition with firms setting prices when they know their own, but not their rivals', cost. He argues that asymmetric information stimulates competition by providing incentives for firms to invest in the set-up costs of entry.

Nonetheless, relative to the first-best outcome that would be chosen by a social planner, in our study the free entry long-run equilibrium under cost asymmetry exhibits a lower degree of excessive entry. ${ }^{1}$ Furthermore, in the welfare analysis we show that cost heterogeneity determines a production allocative inefficiency, which does not arise in the homogeneous firms' case. Indeed due to the weighting system, highly (in)efficient firms find it optimal to increase (decrease) their prices so that (in)efficient firms end up with a smaller (larger) market share.

In the second entry game under study, the dynamics of entry is split into two stages. First, firms have to pay an entry fee in order to learn their production cost realization, but not that of their rivals; second, since firms with a high cost draw may not survive the competition of a highly efficient neighbor, a selection stage determines the firms that can profitably pay a fixed fee in order to enter the production stage. Such a set-up, complemented with the full-information price competition stage, shares with Nocke (2006), Melitz and Ottaviano (2008) and Syverson (2004) the result that larger markets select more efficient firms, i.e., from an empirical point of view denser markets should be characterized by lower inefficiency and price dispersion. Furthermore, we find the selection mechanism that eliminates the least efficient firms becomes more stringent as the variance of the costs among all potential producers increases. On the whole, in the more realistic case where firms have different costs and decide whether to enter under uncertainty, our results point at market outcomes with better welfare properties than those characterizing the simpler symmetric case.

To introduce heterogeneity within the basic circular market structure, this paper adopts a very recent approach, which maintains and extends the original Salop's setup to cope with asymmetric firms. Indeed, our work is related and complements that of Vogel (2008), which considers a two-stage game in which firms first choose their locations and then compete in price. In both stages firms have full information about their own and their rivals' costs and locations, while entry in this paper occurs under uncertainty. Vogel's model has an interesting implication: that lower-cost firms are more isolated in equilibrium. The basic idea is that higher-cost firms try to locate themselves further away from their lower-cost competitors to shield themselves from competition. While the analogies

\footnotetext{
${ }^{1}$ See also Creane (2007) for a model where uncertainty may lead to insufficient entry.
} 
with our set-up are mainly on the pricing game, the interests of the two papers are quite different, since we extensively discuss the welfare properties of two long-run equilibrium models.

The other approach followed in the literature includes studies suggesting changes to either the supply or the demand side of the original set-up. In Bouckaert (2000), firms can either operate a (traditional) retail store located on the circle or set up a mail order business operating from the centre and competing with the traditional store in a nonlocalized way. The presence of the latter intensifies competition so that a smaller number of firms are active in equilibrium compared to the Salop's original model. ${ }^{2}$ To augment the standard circular city model, Anderson and de Palma (2000) use a logit specification for consumers' tastes to combine spatial location, i.e., localized competition, and consumers' love for variety (which introduces an element of global competition): their interaction yields a rich set of qualitative results that can be interpreted in terms of the evolution of industrial structure over the last two centuries. ${ }^{3}$ Von Ungern-Sternberg (1991) presents a model of monopolistic competition where the consumers are located along the edges of a pyramid and not along a circle. The advantage of this approach is that it allows for non-localized competition among firms with different production costs, while, at the same time, it retains some of the features of the circular model. ${ }^{4}$

The next Section describes the model's set-up and characterizes the equilibrium prices as well as the condition for a unique Nash equilibrium in pure strategies. It is followed by the analysis of the market equilibrium, which is then compared, in Section 4, with the socially optimum outcome. The three-stage entry game with a selection mechanism is developed in Section 5. Section 6 concludes. The Appendix contains the proofs for all the Propositions, Lemmas and Corollaries not given in the text.

\footnotetext{
${ }^{2}$ The social inefficiency associated with excessive entry is also mitigated, but not completely eliminated, when firms can price discriminate, both perfectly and imperfectly (Baskar and To, 2004; Liu and Serfes, 2005).

${ }^{3}$ For instance, (i) a decrease in transport costs is expected to lead to a long-run equilibrium with fewer and larger firms, and lower prices and; (ii) an increase in population, and hence in market density, determines a less than proportional increase in the number of firms, so that more product varieties are available to consumers living in larger cities but; (iii) holding market density constant, the same increase in population determines that less effective variety is available in larger cities. Our model generates predictions similar to (i) and (ii); see Nocke (2006) for a result along the same lines as (iii).

${ }^{4}$ More recently, Chen and Riordan (2007) have proposed the "spokes" model as a another way to introduce non-localized competition in a spatially differentiated oligopolistic market.
} 


\section{The model}

Consider a circular city of unitary length with uniform density $D$. There are $N \geq 2$ equidistant firms, ${ }^{5}$ whose location is denoted by the sequence of integers:

$$
n \in \mathbb{L}=\left\{\underline{l}(N)=\left\lceil\frac{1-N}{2}\right\rceil,\left\lceil\frac{1-N}{2}\right\rceil+1, \cdot \cdot, 0,1, \cdot \cdot \bar{l}(N)=\left\lceil\frac{N-1}{2}\right\rceil\right\}
$$

where $\lceil x\rceil$ is the approximation of $x$ to its larger integer. ${ }^{6}$ As a convention and without loss of generality, the chosen notation for firms' location implies that after attributing the value $\bar{l}$ randomly, all the other indexes are assigned in descending order by moving clockwise (see Figure 1 for an example with $N=12$ ). $n$ unambiguously denotes a firm's location, and therefore constitutes an absolute index.

Unit variable costs are $c_{n} \geq 0$ with $c_{n} \in\left[c_{L}, c_{H}\right]$. Thus, we allow firms to have different costs; this may be due to differing managerial abilities across firms (Bartelsman and Doms, 2000), to location-specific factors (Aiura and Sato, 2008) or to different costs in producing varieties (Montagna, 1995; Waterson, 1990). Throughout the paper we assume that firms compete by setting their price when costs and locations are common knowledge.

The short-run number of firms, $N$, is given, e.g., due to entry barriers. Denote the number of locations separating any two firms as $i \in \mathbb{L}$. For firm $n, i<0$ identifies the firm which is its $i$-step clockwise neighbour, while $i>0$ refers to firm $n$ 's $i$-step counterclockwise neighbour. Let \langle\rangle identify an operator such that $\langle n+i\rangle \in \mathbb{L}$ and:

$$
\langle n+i\rangle= \begin{cases}n+i & \text { if } \underline{l} \leq n+i \leq \bar{l} \\ n+i-N & \text { if } n+i>\bar{l} \\ n+i+N & \text { if } n+i<\underline{l}\end{cases}
$$

Consumers incur linear unit transport costs $t$, and have unitary inelastic demand. Consider a consumer who is located at distance $d \in[0,1 / N]$ from firm $n$. The utility she obtains from buying the product from firm $n$ is $U_{d, n}=v-t d-p_{n}$, where $v$ is a positive constant, and $p_{n}$ is the uniform price charged by firm $n$. We assume that the reservation value $v$ is sufficiently high so that consumers buy the good in equilibrium.

\footnotetext{
${ }^{5}$ Throughout the paper we assume sufficiently high relocation costs to prevent a firm to want to change its location, once it has been taken.

${ }^{6}$ That is, if $N=12$, then $\underline{l}(12)=-5$ and $\bar{l}(12)=6$, but if $N=11$, then $\underline{l}(11)=-5$ and $\bar{l}(11)=5$. For notational convenience, the dependence of $\underline{l}$ and $\bar{l}$ on $N$ will not be carried explicitly.
} 


\subsection{Equilibrium prices}

Condition 1 (No mill-price undercutting) $\left|p_{n}-p_{\langle n+1\rangle}\right|<\hat{k}$, for $n \in \mathbb{L}$, where $\hat{k}=t / N$.

Following Eaton and Lipsey (1978), we keep the requirement that competition is localized: the market share and price setting of a firm located in $n$ is directly affected only by the behaviour of the two adjacent firms. That is, firms do not reduce prices in such a way to grab all the market of one or both 1-step neighbours. However, satisfying Condition 1 does not necessarily produce a Nash equilibrium in prices because a highly efficient firm could undercut and drive a highly inefficient 1-step neighbour from the market. We will now characterize the analytical solution for the equilibrium prices, leaving the derivation of the conditions on costs under which the Nash price equilibrium exists to the next sub-section.

Standard computations yield firm n's demand (Tirole, 1988, p. 283):

$$
q_{n}=D \cdot\left(\frac{1}{N}+\frac{p_{\langle n-1\rangle}+p_{\langle n+1\rangle}-2 p_{n}}{2 t}\right), n \in \mathbb{L} ;
$$

firm $n$ 's profit function is thus: $\Pi_{n}=\left(p_{n}-c_{n}\right) \cdot q_{n}$. Concavity of the profit function in $p_{n}$ implies that the optimal prices for the $N$ firms are given by the first order conditions:

$$
p_{n}=\frac{2 c_{n}+p_{\langle n-1\rangle}+p_{\langle n+1\rangle}}{4}+\frac{1}{2} \hat{k}, \forall n \in \mathbb{L} .
$$

Lemma 1 If Condition 1 is satisfied, then system (2) has a unique solution:

$$
p_{n}^{*}=\sum_{i=\underline{l}}^{\bar{l}} w_{\bar{l}-|i|} c_{\langle n+i\rangle}+k, n \in \mathbb{L} .
$$

Although competition is localized, Lemma 1 highlights the inter-connection of all firms' pricing strategies, an aspect that does not feature in a symmetric costs set-up. Consider the firm in $n=0$ (Figure 1 ). From (2), the price charged by firm 0 depends on its costs and on the prices charged by its adjacent firms. The latter's prices depend on their own costs and by the price charged by, respectively, firm 0 and their respective other 1-step neighbour. Similarly, the price charged by the 2 -step neighbours of firm 0 depends on their own costs and on the price charged by their 1-step neighbours, and so on and so forth. In other words, despite the localized competition assumption, the total market is the result of chain-linked sub-markets (Chamberlin, 1956, p. 102; Rothchild, 1982), so that each firm's cost influences the pricing of the non-direct competitors through a domino effect. The degree of interconnection between firms' costs and prices is captured by the set of weights $w_{i}$, while $k$ is an average mark-up reflecting the degree of substitutability across 
products (as in standard homogeneous cost models).

It is worth noting that the adopted conventional notation assigns the weight with the highest subscript to a firm's own cost, and that, for even $N, w_{0}$ corresponds to the weight for the cost of the firm which is opposite (i.e., furthest) to firm 0 (see Figure 1); for an odd $N, w_{0}$ is the weight for both firms $\underline{l}$ and $\bar{l}$. Interestingly, the weights are not influenced by the cost differential between firm $n$ and its $i$-step neighbours. That is, each firm in the market assigns the same weight to the cost of a firm regardless of the level of that cost. Furthermore, due to the domino effect the weights are also expected to decrease with respect to distance, that is, $w_{0}<w_{1}<. .<w_{\bar{l}}$.

Proposition 1 (Characterization) Assume the existence of a Nash price equilibrium, which satisfies Condition 1; then for any $N \geq 2$, the solution is unique and it is given by (3) of Lemma 1, where the weights $w_{i}$ and $k$ are:

$$
\begin{aligned}
& w_{i}=4 w_{i-1}-w_{i-2} \text { for } i=1, . . \bar{l}-1 \\
& w_{1}=(3-\underline{l}-\bar{l}) w_{0} \\
& 4 w_{\bar{l}}=2+2 w_{\bar{l}-1} \\
& \sum_{i=\underline{l}}^{\bar{l}} w_{\bar{l}-|i|}=1, \\
& k=\hat{k}=t / N .
\end{aligned}
$$

(Sketch of the proof.) While a formal proof is shown in the Appendix, here we provide the intuition behind the derivation of (4) and (5) assuming $N$ is even. From Lemma 1, firm 0's equilibrium strategy is of the form:

$$
p_{0}=w_{\bar{l}} c_{0}+w_{\bar{l}-1}\left(c_{-1}+c_{+1}\right)+w_{\bar{l}-2}\left(c_{-2}+c_{+2}\right)+\ldots+w_{0} c_{\bar{l}}+k .
$$

By the same token, using the shift in weights' notation described in Figure 1, the price charged by the adjacent firms located at +1 and -1 , respectively, are:

$$
\begin{aligned}
& p_{-1}=w_{\bar{l}} c_{-1}+w_{\bar{l}-1}\left(c_{-2}+c_{0}\right)+w_{\bar{l}-2}\left(c_{-3}+c_{+1}\right)+\ldots+w_{0} c_{\bar{l}-1}+k \\
& p_{+1}=w_{\bar{l}} c_{+1}+w_{\bar{l}-1}\left(c_{0}+c_{+2}\right)+w_{\bar{l}-2}\left(c_{-1}+c_{+3}\right)+\ldots+w_{0} c_{\underline{l}}+k .
\end{aligned}
$$

Now substituting (6), (7) and (8) in (2), with $n=0$, and collecting the similar terms, we obtain:

$$
\begin{gathered}
c_{0}\left(4 w_{\bar{l}}-2-2 w_{\bar{l}-1}\right)+\left(c_{-1}+c_{1}\right)\left(4 w_{\bar{l}-1}-w_{\bar{l}-2}-w_{\bar{l}}\right)+\left(c_{-2}+c_{2}\right)\left(4 w_{\bar{l}-1}-w_{\bar{l}}-w_{\bar{l}-2}\right)+. . \\
. .+\left(c_{\underline{l}}+c_{\bar{l}-1}\right)\left(4 w_{1}-w_{0}-w_{2}\right)+c_{\bar{l}}\left(4 w_{0}-2 w_{1}\right)+(2 k-2 \hat{k})=0 .
\end{gathered}
$$


In order for this equality to hold for every possible configuration of $c_{n}, n \in \mathbb{L}$, all the expressions in round brackets must be equal to zero, thereby proving (4a)-(4c) and (5). Finally, (4d) emerges by replacing $c_{n}=c$ for $n \in \mathbb{L}$ in (9). ${ }^{7}$

Given (4d), when firms have identical costs $c_{n}=c, \forall n \in \mathbb{L}$, (3) reduces to the standard solution in Salop (1979): $p_{n}=c+\hat{k}, \forall n$.

Proposition 1 highlights the relationship among the weights $w_{i} \mathrm{~s}$ in Lemma 1 ; however finding their numerical values using (4) can be computationally burdensome, because it would be necessary to solve a system of $\lceil(N+1) / 2\rceil$ equations. We now provide a simpler way to compute them.

Corollary 1 Let:

$$
\begin{aligned}
a(i) & =4 a(i-1)-a(i-2), \text { for } i=2, . . \bar{l}, \\
a(0) & =1, a(1)=3-\underline{l}-\bar{l}, \text { and } \\
A_{N} & =\sum_{i=\underline{l}}^{\bar{l}} a(\bar{l}-|i|) .
\end{aligned}
$$

Then

$$
w_{i}=a(i) / A_{N}
$$

The recursive nature of (10) facilitates the computation of the weights given the initial conditions (10b). Corollary 1 is used in Table 1 to obtain the numerical values of the optimal weights for $N=2,3, . ., 15,20, \infty$.

\section{[Insert Table 1]}

From Table 1 one can observe that for $N>7$, the weights associated to a firm's own cost and to those of the three closest neighbours are, respectively, about 57.7\%, 15.5\%, $4.2 \%$ and $1.4 \%$, and that such weights remain almost constant as $N$ increases. For $N \geq 8$, the costs of the firms that are farther than 3 steps have a highly negligible impact on the price set by some other firm in the market $\left(R=1-\sum_{i=\max (-3, \underline{l})}^{\min (3, \bar{l})} w_{\bar{l}-i}\right.$ is less than $\left.1 \%\right){ }^{8}$ Therefore, given the weights' high speed of convergence, even for a relatively small $N$ the following limiting result can be used to derive a good approximation of equilibrium prices.

Corollary 2 When $N \rightarrow \infty$, for every $i$, then $w_{\bar{l}} \rightarrow y=1 / \sqrt{3} ; w_{\bar{l}-i} / w_{\bar{l}-i-1} \rightarrow x=$ $2+\sqrt{3} ; w_{\bar{l}-i} \rightarrow y \cdot x^{-i}$.

\footnotetext{
${ }^{7}$ Strictly speaking, only two conditions among (4b), (4c) and (4d) are necessary to derive all the weights.

${ }^{8}$ Pinkse et al. (2002) analyze competition among wholesalers of oil refined products by using a number of closeness measures to proxy for the degree of product differentiation: only the coefficient for the nearest neighbor rival price is significant and economically meaningful. Their estimates reveal that direct rivalry decays abruptly with distance, suggesting that competition is highly localized.
} 


\subsection{Price equilibrium existence and uniqueness}

We now show the existence of the equilibrium in Proposition 1 under two related firms' behavioural assumptions; in the first firms commit themselves to Condition 1, in the second this same condition holds without any commitment on the firm's part. In both cases we need to impose some restrictions on the cost differential between two neighbouring firms either because otherwise the no-mill price condition would fail to hold, or because the efficiency gap may be such that a highly efficient firm can profitably undercut a 1-step inefficient neighbour and drive it from the market.

Proposition 2 (Existence and Uniqueness under firms' commitment to Condition 1) Equations (3)-(5) represent a unique Nash price equilibrium in pure strategies, if the maximum cost differential is not too large relative to the degree of product differentiation:

$$
c_{H}-c_{L}<\rho_{c}(N) \hat{k},
$$

where $\hat{k}=t / N$ and $\rho_{c}(N)=\left(w_{\bar{l}}-w_{0}\right)^{-1}$.

Although largely used in the literature, assuming a commitment to the no-mill price undercutting condition is restrictive because it does not generally lead to undercut-proof prices. Without commitment, the efficiency gap may be such that a highly efficient firm can profitably undercut a 1-step inefficient neighbour and drive it from the market unless we impose a restriction stronger than (12).

Proposition 3 (Existence and Uniqueness) Equations (3)-(5) represent a unique Nash price equilibrium in pure strategies, if the maximum cost differential is not too large relative to the degree of product differentiation:

$$
c_{H}-c_{L}<\rho(N) \hat{k},
$$

- $\rho(2)=\rho_{c}(2)=3$;

- $\rho(N)=\frac{1}{A}\left(B-\sqrt{B^{2}-A}\right)$ for $N \geq 3$,

$$
\begin{aligned}
& A=\left(1-w_{l-1}\right)^{2}-\left(1-w_{l}\right)^{2}-\left(1-w_{l-1}\right)\left(w_{l}-w_{l-2}\right), \\
& B=\left(1-w_{l-1}\right) ;
\end{aligned}
$$

- $\lim _{N \rightarrow \infty} \rho(N)=\bar{\rho} \simeq 0.60974$. 
First, when $N=2$ firms have no incentive to undercut the rivals, because $\rho(2)=$ $\rho_{c}(2)=3$. That is, the equilibrium in two-firm Hotelling type game is always undercutproof (Hamilton et al., 1991).

Second, the values of $\rho_{c}$ and $\rho$ as a function of $N$ are reported in Table 2. Clearly, for $N \geq 3, \rho_{c}$ is about three times greater than $\rho$ because an under-cut proof equilibrium is incompatible with excessive cost heterogeneity. Many authors have noted that, in horizontal product differentiation models, the non-existence of an equilibrium in pure strategies is due to the small degree of product differentiation (d'Aspremont et. al., 1979). ${ }^{9}$ Similarly, in our case, as $N$ increases conditions (12) and (13) become more stringent because $\hat{k}=t / N, \rho_{c}$ and $\rho$ decrease in $N$.

Finally, both Propositions provide a sufficient condition for the existence and uniqueness of the Nash equilibrium in pure strategies which hold under every possible costs' structure. Different assumptions on the latter would generally lead to a less stringent specification of conditions (12) and (13). For instance, if the costs of two neighbouring firms are positively correlated (e.g., because costs depend on the distance from an input source), then intuitively a Nash equilibrium would still exist even if the constraint on $c_{H}-c_{L}$ were relaxed.

\section{Long-run equilibrium}

Suppose the number of firms is not given, but that it is endogenously determined. Following Salop (1979), in this section we consider a simple two-stage entry game with the entry stage followed by the production stage. In the first stage, those potential entrants choosing to enter have to pay a set-up cost $F$, the others remain out of the market and gain zero profit. Before paying $F$, each potential entrant only knows the costs distribution but has no information on the actual realization of its own and its potential opponents' costs. ${ }^{10}$ After investing $F$ and occupying a random equidistant location, in the production stage competition takes place among the $N$ entrants as in Section 2, so that locations and costs are common knowledge. We solve the model by backward induction. The price equilibrium in the production stage is therefore given by Propositions 1 under the existence conditions of Propositions 2 and 3.

\footnotetext{
${ }^{9}$ An equilibrium in mixed strategies has been proved to exist for the Hotelling linear city case (Dasgupta and Maskin, 1986).

${ }^{10}$ This is an important difference with Vogel (2008), which nonetheless shows that symmetric locations would be chosen by firms with same costs. Intuitively, under full uncertainty, firms are in expectation all ex-ante identical and would therefore choose, as we assume, to locate equidistantly.
} 


\subsection{Entry phase}

To derive the properties of the long-run equilibrium, we assume that costs are identically and independently distributed:

Assumption 1 Let $\tilde{c}_{n} \in\left[c_{L}, c_{H}\right]$ be a random variable with distribution $G$, mean value $E\left[c_{n}\right]=\bar{c}$, variance $E\left[\left(c_{n}-\bar{c}\right)^{2}\right]=\sigma^{2}$ and covariance $E\left[\left(c_{n}-\bar{c}\right) c_{m}\right]=0, \forall n, m \in \mathbb{L}$ and $n \neq m$.

Firms are risk-neutral and have perfect foresight, so that they enter the market if their expected profit is non-negative. Since costs are unknown in the first stage, firm $n$ 's random profit is:

$$
\left.\tilde{\Pi}_{n}=\frac{D}{t}\left(\tilde{p}_{n}-\tilde{c}_{n}\right) \cdot \frac{1}{2}\left(\tilde{p}_{\langle n-1\rangle}+\tilde{p}_{\langle n+1\rangle}\right)-\tilde{p}_{n}+\hat{k}\right)-F,
$$

where the superscript $\sim$ denotes stochastic variables.

Proposition 4 The expected profit of firm $n$ is:

$$
E\left[\tilde{\Pi}_{n}\right]=\frac{D}{t}\left(\sigma^{2} W_{I}(N)+\hat{k}^{2}\right)-F
$$

- $W_{I}(N)=\sum_{i=\underline{l}}^{\bar{l}} w_{\bar{l}-|i|}\left[\left(w_{\bar{l}}-w_{\bar{l}-1}\right)-\left(w_{\bar{l}-|i|}-w_{\bar{l}-|i+1|}\right)\right]>0$, with $w_{-1}:=w_{\bar{l}+\underline{l}}$;

- $\lim _{N \rightarrow \infty} W_{I}(N)=\bar{W}_{I}=1-\frac{4}{9} \sqrt{3} \simeq 0.23020$.

The values of $W_{I}(N)$ are reported in Table 2 : they monotonically decrease for $N \geq 3$, and rapidly converge toward $\bar{W}_{I}$. From Proposition 4 , the expected profit $E\left[\tilde{\Pi}_{n} \mid N\right]$ decreases in $N$ and $F$ and increases in $D, \sigma$ and $t{ }^{11}$ An interesting result is that the expected profits increase with cost heterogeneity. This is due to the profit function being convex in $c_{i}$ so that, for the Jensen inequality, $E(\Pi(\tilde{c}))>\Pi(E(\tilde{c}))$ (Spulber, 1995). For instance, in the extreme case where any two adjacent firms' costs are perfectly negatively correlated, i.e., $E\left[\left(c_{n}-\bar{c}\right) c_{\langle n+1\rangle}\right]=-\sigma^{2}$ and $\mathrm{N}$ is even, it can be shown that the expected profit (15) reduces to $E[\Pi]=\frac{D}{t}\left(\hat{k}^{2}+\frac{4}{9} \sigma^{2}\right)-F .^{12}$ That is, firms prefer to "gamble" even if there is a fifty-fifty chance of drawing a high cost. Furthermore, when there is perfect

\footnotetext{
${ }^{11}$ Taking the derivative of $E\left[\tilde{\Pi}_{n} \mid N\right]$ with respect to $t$, we obtain that the expected profit is increasing in $t$ when $\sigma<\hat{k} / \sqrt{W_{I}} \simeq 2.09 \hat{k}$. In the support $\left[c_{l}, c_{h}\right]$ the maximal variance arises from a distribution where all the mass is equally concentrated on the extremes: $\sigma=\left(c_{h}-c_{l}\right) / 2$; hence, under (12) and (13) the expected profit is always increasing in $t$ for any distribution.

${ }^{12}$ Perfect negative correlation is obtained when the 1-step neighbors of a $c_{l}$-type firm are $c_{h}$-types, and viceversa. Calculations are available on request.
} 
positive pairwise correlation among costs, $\sigma^{2}=0$ and the profits are the same as the case of no uncertainty (i.e., Salop).

Proposition 5 From Proposition 4, the equilibrium number of firms in the market, $N_{M}$, is implicitly given by the following equation:

$$
D \cdot\left(\frac{t}{N_{M}^{2}}+\frac{\sigma^{2}}{t} W_{I}\left(N_{M}\right)\right)=F
$$

For $N$ large, the number of firms is approximated by:

$$
N_{M} \simeq \sqrt{\frac{t}{F / D-\bar{W}_{I} \sigma^{2} / t}}
$$

It is noteworthy that $N_{M}$ converges to the value in Salop (1979) when firms' costs are identical, i.e., $N_{M}=\sqrt{t D / F}$ when $\sigma^{2}=0$. More importantly, the game with cost heterogeneity yields a higher number of firms in equilibrium, relative to that in the traditional case. However, the two cases are not perfectly comparable given the different informational structure they assume. Therefore, in order to gauge the extent to which the free-entry outcome is excessive, we need to establish how the free-entry equilibrium under cost heterogeneity differs from the socially optimum outcome.

\section{Welfare analysis}

In the spirit of Salop (1979), in this Section we compare the free-entry market equilibrium in Proposition 5 with the first-best optimum that would be chosen by a social planner. It is worth stating up-front that relative to the welfare analysis in Salop (1979), in the present case we have to consider how the socially optimal definition of each firm's market share impacts on the total production cost incurred in the economy. This is tantamount to assessing whether the market equilibrium pricing rules induce allocative inefficiency so that consumers who should patronize a low-cost firm end up buying from a higher cost firm.

\subsection{Production}

Assume there are $N$ firms already settled on the circle, with a generic firm $n$ having $\operatorname{cost} c_{n} \in\left[c_{L}, c_{H}\right]$. We focus on the generic arc of length $\frac{1}{N}$ defined by the locations of firms $\langle n-1\rangle$ and $n$. Because demand is inelastic, the first-best allocative solution is reached when the sum of production and transport costs is minimized. The solution to 
this problem, which is worked out when the planner has learnt the cost realization in each location, is equivalent to finding the optimal allocation of consumers between two adjacent firms. However, unlike the standard case, where the planner splits the arc evenly among firms, optimality under cost heterogeneity may require that the most efficient firms supply a larger proportion of the market even if this increases transport costs.

Let $d_{n}$ and $d_{\langle n-1\rangle}=\left(\frac{1}{N}-d_{n}\right)$ be the distance of the pivotal consumer from two adjacent firms. The average distance between a generic consumer on the arc and the seller she patronizes is:

$$
\bar{d}=N\left(\int_{0}^{d_{\langle n-1\rangle}} x d x+\int_{0}^{d_{n}} x d x\right)=\frac{N}{2}\left(d_{\langle n-1\rangle}^{2}+d_{n}^{2}\right) .
$$

Hence, the average transport costs among all consumers on the arc is:

$$
T C=\bar{d} t=\frac{N}{2}\left(d_{\langle n-1\rangle}^{2}+d_{n}^{2}\right) t
$$

The proportion of consumers patronizing firm $\langle n-1\rangle$ and firm $n$ are, respectively, $N d_{\langle n-1\rangle}$ and $N d_{n}$. The average (variable) cost of producing goods for a consumer on the arc is:

$$
V C=N d_{\langle n-1\rangle} c_{\langle n-1\rangle}+N d_{n} c_{n}
$$

The social planner problem can be expressed as:

$$
\min _{d_{n} \geq 0}(T C+V C)=\frac{N}{2}\left(\left(\frac{1}{N}-d_{n}\right)^{2}+d_{n}^{2}\right) t+N\left(\frac{1}{N}-d_{n}\right) c_{\langle n-1\rangle}+N d_{n} c_{n}
$$

Therefore,

$$
d_{n}^{*}=\frac{1}{2 N}+\left(\frac{c_{\langle n-1\rangle}-c_{n}}{2 t}\right) .
$$

Because $d_{n}^{*} \geq 0$, then (21) holds if $c_{H}-c_{L}<\hat{k}$.

The implementation of (21) can be obtained by using prices as policy instruments. From:

$$
d_{\langle n-1\rangle}=\frac{1}{2 N}+\frac{p_{n}-p_{\langle n-1\rangle}}{2 t} ; d_{n}=\frac{1}{2 N}+\frac{p_{\langle n-1\rangle}-p_{n}}{2 t}
$$

we obtain: 
Lemma 2 The first-best full information allocative solution can be obtained by setting:

$$
p_{n}^{F}=c_{n}+k_{F},
$$

where $k_{F}$ can be set freely with the only caution that the delivered price does not exceed the reservation price of consumers.

Comparing (3) and (23), it immediately follows that if firms have heterogeneous costs, for any given $N$, the market prices determine a distortive allocative effect. Indeed, when firms have heterogeneous costs their optimizing behavior induces them not only to base their prices on their own costs (as in the first best situation) but also on those of the opponents. That is, for given $k$, efficient firms charge prices above the first best solution and inefficient ones generally charge prices below it.

\subsection{Entry}

In Section 3.1 the decision on entry is made under uncertainty. Equivalently, here we study the planner's long-run choice using Assumption 1, so that the first-best optimal number of firms is such that it minimizes the expected sum of transport, production and fixed costs.

Using (18), (19), (21) and Assumption (1), the expected average transport cost $E[T C]$ and the expected average (variable) cost of production $E[V C]$ are:

$$
E[T C]=\frac{t}{4 N}+\frac{1}{2} \frac{N}{t} \sigma^{2} ; E[V C]=\bar{c}-\frac{N}{t} \sigma^{2} .
$$

Finally, fixed costs per consumer are: $F C=N F / D$. Hence, the expected average total cost:

$$
E[C]=E[T C+V C+F C]=\left(\frac{t}{4 N}+\frac{1}{2} \frac{N}{t} \sigma^{2}\right)+\left(\bar{c}-\frac{N}{t} \sigma^{2}\right)+\frac{N F}{D}
$$

Proposition 6 The first-best optimal number of firms is:

$$
N_{F}=\frac{1}{2} \sqrt{\frac{t}{F / D-0.5 \sigma^{2} / t}}
$$

Discussion. Note from (24) that an increase in cost heterogeneity, captured by $\sigma$, increases transport costs less than it reduces the production costs because the planner can shift production towards the more efficient plants. Thus, our analysis highlights a welfareenhancing effect of cost heterogeneity, whose overall impact has to be investigated within the limits imposed by Propositions (2) and (3). Indeed, for any $G$ with support $\left[c_{L}, c_{H}\right]$ 
it must be that $\sigma \leq\left(c_{H}-c_{L}\right) / 2$ and therefore, given that $\rho_{c}>\rho$, the condition on (12) allows the analysis of cases with larger allowable cost diversity than under condition (13). ${ }^{13}$

With regards to the socially optimal number of firms, from (16), (25) and $\bar{W}_{I}<0.5$, we have that $N_{M} / N_{F}<2$ when $\sigma>0$, while this ratio is equal to 2 when firms' costs are identical (Salop, 1979). The condition (12) determines a lower bound for $N_{M} / N_{F}$. Numerical simulations run imposing $0 \leq \sigma \leq\left(c_{H}-c_{L}\right) / 2=(0.16) / 2$ show that $N_{M} / N_{F}$ is indeed monotonically decreasing in $\sigma$ and reaches a minimum value around $1.78 .{ }^{14}$ That is, the greater the degree of heterogeneity, the less excessive market entry is.

In terms of the overall costs (24), we can compare market efficiency under different regulatory scenarios. To emphasize the role of cost heterogeneity, our simulation analysis assumes the maximum value of costs' variance satisfying condition (12). Figure 2 shows the relationship between the number of firms and the expected average total cost $E[C]$ under three different pricing schemes: uniform (the regulator imposes the same price for all firms; this is equivalent to the case where $c_{n}=\bar{c}$ for all $n$ ), market (prices are set according to Proposition 1), first-best (the marginal cost pricing in (23)). ${ }^{15}$ The same Figure reports the first-best number of firms as well as the long-run equilibrium under free entry. Obviously, the first-best solution in $F$ has the highest level of welfare, but its implementation requires the gathering of information on each firm's cost. It also implies having consumers that pay different mill prices, which may not be a politically viable strategy. Nonetheless, the uniform pricing outcome in $U$ is highly inefficient relative to $F$. The expected total social cost in point $U$ is slightly lower than in $M$, but the latter does not require any regulatory intervention. Relative to the uniform case, some welfare gains can be achieved if the planner decides to regulate entry by moving the market outcome from $M$ to $S$, the latter being closer to $F$ than to $U$. To conclude, when the implementation of the first-best outcome is infeasible, in the presence of large cost heterogeneity the market outcome is almost welfare-equivalent to the outcome that would arise when a regulator combines a uniform price with entry restrictions. ${ }^{16}$ Welfare-

\footnotetext{
${ }^{13}$ For instance, when $G$ is uniform $\sigma=\left(c_{h}-c_{l}\right) / 2 \sqrt{3}$, but when all the mass is equally concentrated on the extremes: $\sigma=\left(c_{h}-c_{l}\right) / 2$.

${ }^{14}$ The other values were: $F=t=1, D=100, \bar{c}=0.1$. When the same simulation was run under condition (13) the lower bound was about 1.97. Note however, that smaller lower bounds could have been achieved by explicitly dealing with the integer problem.

${ }^{15} E[C]$ can be expressed more succinctly in terms of the pricing scheme using the following:

$E[C]=\frac{1}{4} \hat{k}+\bar{c}+N F / D-\sigma^{2} \hat{k}^{-1} \chi$, where $\chi=0$ under uniform pricing, $\chi \simeq 0.65$ under market pricing, and $\chi=1$ under first-best pricing.

${ }^{16}$ The econometric evidence in Schaumans and Verboven (2006) on the Belgian pharmacies market provides support to this argument: the current regime with restricted entry and high regulated markups seems to protect the private interests of pharmacies. Its replacement with a new regime with free entry and reduced markups appears likely to generate substantial cost savings to consumers without the risk of reducing the availability of supply.
} 
improvements should be therefore achieved not by regulating prices, but by limiting entry, which leaves open the question of how the regulator can induce entry of the most efficient potential entrants. In the next Section we present an entry game where such a selection is endogenously determined within a market mechanism.

\section{Long-run equilibrium with selection}

In this Section we consider a more sophisticated three stage entry game, which includes a pre-entry and a selection stage; in the latter, a firm's decision to enter in the production (price competition) stage depend on the cost drawn in the previous stage. The timing is as follows.

Stage 1 - Pre-Entry; $M$ firms invest $F_{e}$ and learn their own cost but not that of their rivals; they anticipate that in the selection phase $M-N$ firms will not continue;

Stage 2 - Selection; Based on their own cost and on the belief on the rival's cost, firms decide whether to stay or exit the market; the remaining firms are those whose costs are below a certain threshold; they anticipate that the pricing stage will be played according to the Proposition 1;

Stage 3 - Full Information Pricing; The remaining $N$ firms incur a fixed set-up cost $F_{p}$ and set their prices under a full information scenario; i.e., they know their rivals' costs.

When $F_{e}=F$ and $F_{p}=0$, we go back to the previous long-run equilibrium discussed in Section 3. The timing reflects the empirical evidence suggesting that firms do actually sink resources before learning their costs (as in Stage 1) and that selection constitutes an essential element in competitive industries, which is for instance revealed by higher exit rates for young plants (Dunne et al., 1989; Foster et al., 2006).

There is an important difference between the structure of our game and that presented in Syverson (2004) where firms set their prices based only on their cost type but not on their rivals' actual cost realizations. While this may be a realistic assumption in the shortrun, our approach closely mimics a complete information, long-run equilibrium where firms have learnt to set prices from which they would not unilaterally want to deviate (which, given Proposition 1, implies a full knowledge of all the firms' costs and locations as in Vogel (2008)).

We solve the model by backward induction. The firms' profits in stage 3 are computed using Proposition 1. Throughout the Section, restrictions (13) or (12) on maximal cost heterogeneity are maintained. 


\subsection{Selection}

In the second stage, a generic firm $n$ knows $c_{n}, M$ and the prior distribution of costs $G$. The assumptions on the distribution of $G$ are given by Assumption 1. Because costs are randomly and independently distributed, learning its own cost does not change a firm's beliefs on its rivals' costs. Nonetheless, in the selection phase firms can form their beliefs as to the cost distribution that will emerge in the price competition stage, where only a subset $N \leq M$ of firms may be involved. Based on such beliefs, each firm assumes a posterior distribution of the opponents' costs $G_{e}$, which reflects each firm's beliefs on its rivals' decision on whether to stay or exit the market.

Under the assumption that there is at least one opponent that is going to enter the market, the expected profit (gross of pre-entry fee $F_{e}$ ) of firm $n$, if it enters the market (together with $N-1 \geq 1$ other competitors) is:

$$
\begin{aligned}
E_{G_{e}}\left[\tilde{\Pi}_{n} \mid c_{n}, N, M\right] & =\frac{D}{2 t} \cdot E_{G_{e}}\left[\left(\tilde{p}_{n}-c_{n}\right) \cdot\left(\tilde{p}_{\langle n-1\rangle}+\tilde{p}_{\langle n+1\rangle}-2 \tilde{p}_{n}+2 \hat{k}\right)\right]-F_{p} \\
& =\frac{D}{t} \cdot E_{G_{e}}\left[\left(\tilde{p}_{n}-c_{n}\right) \cdot\left(\tilde{p}_{\langle n-1\rangle}-\tilde{p}_{n}+\hat{k}\right)\right]-F_{p}
\end{aligned}
$$

where $E_{G_{e}}$ means that the expectations are taken using firm $n$ 's expected cost distribution on entrants, $G_{e}$. The second line in (26) derives from the fact that in stage 2 firm $n$ treats $\tilde{p}_{\langle n-1\rangle}$ and $\tilde{p}_{\langle n+1\rangle}$ in an identical manner. Further, even if firm $n$ is a monopolist, its profit must be bounded from above, since it is limited by the consumers' willingness to pay $v$. Therefore $E_{G_{e}}\left[\tilde{\Pi}_{n} \mid c_{n}, 1, M\right]=D\left(v-c_{n}-t / 2\right)-F_{p}<\infty .{ }^{17}$ Define $\bar{c}_{e}=E_{G_{e}}\left(c_{\langle n+i\rangle}\right)$, $\sigma_{e}^{2}=E_{G_{e}}\left(c_{\langle n+i\rangle}\left(c_{\langle n+i\rangle}-\bar{c}_{e}\right)\right), \forall i \neq 0$.

Proposition 7 Under Assumption 1,

a) the expected profit of firm $n$ is given by:

$$
E_{G_{e}}\left[\tilde{\Pi}_{n} \mid c_{n}, N, M\right]=\frac{D}{t}\left(\left(w_{d}\left(\bar{c}_{e}-c_{n}\right)+\hat{k}\right)^{2}+W_{I I}(N) \sigma_{e}^{2}\right)-F_{p}
$$

where $w_{d}=\left(1-w_{\bar{l}}\right)=\left(w_{\bar{l}}-w_{\bar{l}-1}\right), W_{I I}(N)=\sum_{i=\mathbb{L}_{0}} w_{\bar{l}-|i|}\left(w_{\bar{l}-|i+1|}-w_{\bar{l}-|i|}\right)>0, \mathbb{L}_{0} \equiv$ $\mathbb{L} \backslash\{0\}, \lim _{N \rightarrow \infty} W_{I I}(N)=\bar{W}_{I I}=\frac{2}{9} \sqrt{3}-\frac{1}{3} \simeq 0.051567$.

b) $E_{G_{e}}\left[\tilde{\Pi}_{n} \mid c_{n}, N, M\right]$ is decreasing in $c_{n}$ and $F_{p}$, and increasing in $\bar{c}_{e}$ and $D$.

Proposition 7 holds for each firm and for each possible $N$. Thus, sequential rationality leads to the conclusion that each firm expects that the opponents deciding to enter the

\footnotetext{
${ }^{17}$ The case of monopoly can generate problems of existence of equilibria when the market is too small for two firms but large enough for one firm. See for example Levin and Peck (2003) for a detailed analysis on entry with at least two potential entrants.
} 
market are sufficiently efficient, i.e., their cost is not greater than a threshold level $\alpha$. Therefore, the posterior distribution of such firms' costs corresponds to the prior distribution $G(x)$ truncated at $\alpha$. That is, $G_{e}(x)=G_{\alpha}(x)=\min \{G(x) / G(\alpha), 1\}$.

Conjectures on $\alpha \in\left[c_{L}, c_{H}\right]$ also affect the number of firms that participate to the pre-entry stage: $\tilde{N}=\tilde{N}(\alpha, M)$. Since cost distributions are independent, we compute the probability that there are $\eta \leq M$ (including $n$ ) firms whose costs fall under the entry thresold $\alpha$ :

$$
\omega_{\eta}=\operatorname{Pr}(\tilde{N}=\eta)=G(\alpha)^{\eta}(1-G(\alpha))^{M-\eta} .
$$

It follows that if firm $n$ decides to enter the market, provided that in the first stage there are $M$ firms, its expected profit is: ${ }^{18}$

$$
\sum_{\eta=1}^{M} \omega_{\eta} E_{G_{\alpha}}\left[\tilde{\Pi}_{n} \mid c_{n}, \eta, M\right]
$$

For given $c_{n}, M$ and $\alpha,(28)$, by accounting for any possible industry configuration, represents the expected profit in the selection phase. Equivalently, for $M$ large, the central theorem guarantees that $\tilde{N}(\alpha, M) \rightarrow N(\alpha, M)=G(\alpha) M$ since $\omega_{N} \rightarrow 1 ;(28)$ becomes:

$$
E_{G_{\alpha}}\left[\tilde{\Pi}_{n} \mid c_{n}, M\right]=E_{G_{\alpha}}\left[\tilde{\Pi}_{n} \mid c_{n}, G(\alpha) M, M\right]
$$

In the subsequent analysis, we assume $M$ large so that the expected profit can be approximated by equation (29). Note that $E_{G_{\alpha}}\left[\tilde{\Pi}_{n} \mid c_{n}, M\right]$ retains most of the properties of $E_{G_{e}}\left[\tilde{\Pi}_{n} \mid c_{n}, N, M\right]$ in Proposition 7, i.e.

Lemma $3 E_{G_{\alpha}}\left[\tilde{\Pi}_{n} \mid c_{n}, M\right]$ is increasing in $D$ and decreasing in $c_{n}, F_{p}$ and $M$.

Unlike in previous Sections, the comparative static analysis concerning the impact of a change in cost heterogeneity on the expected profit is not straightforward; indeed, the expected profit in (29) is not only affected by $\sigma$ as in (15), but it also depends on $\bar{c}_{\alpha}, \sigma_{\alpha}$ and $G(\alpha)$. That is, a mean-preserving change of the distribution $G^{\sigma}$ in $G^{\sigma}$, with $\hat{\sigma} \neq \sigma$, may produce different results depending on how the probability mass is distributed. One way to study an increase in heterogeneity can be to assume a stretch of the initial distribution $G^{\sigma}$ to obtain $G^{\sigma}$ with $\hat{\sigma}>\sigma$.

Assumption 2 Let $\mathcal{G}=\left\{G^{\sigma}, \sigma \in(0, \hat{\sigma}]\right\}$ is a family of bounded distributions parametrized by $\sigma$, twice continuously differentiable, with differentiable density $g^{\sigma}$, average $\bar{c}$ and variance $\sigma^{2}$, such that $G^{\sigma}(x)=G^{s}\left(\frac{x-\bar{c}}{\sigma}\right)$, where $G^{s}$ has mean value 0 and variance 1 . In

\footnotetext{
${ }^{18}$ When firm $n$ decides to enter the market it has costs lower than or equal to $\alpha$ and expects that the other firms entering the market have costs lower than or equal to $\alpha$.
} 
addition, let $\phi\left(\alpha, c_{n}, M, \sigma\right)=E_{G_{\alpha}^{\sigma}}\left[\tilde{\Pi}_{n} \mid c_{n}, M\right]$, and $\Phi(\alpha, M, \sigma)=\phi(\alpha, \alpha, M, \sigma)$. We require that:

(A) $G \in \mathcal{G}$.

(B) (monotonicity) $\phi\left(\alpha, c_{n}, M, \sigma\right)$ is decreasing in $\alpha$ and $\sigma$.

(C) (single crossing) $\frac{\phi_{\alpha}(\alpha, \alpha, M, \sigma)}{\phi_{y}(\alpha, \alpha, M, \sigma)}<\frac{\phi_{\alpha}\left(\alpha, c_{n}, M, \sigma\right)}{\phi_{y}\left(\alpha, c_{n}, M, \sigma\right)}$ for $y=M, \sigma$ and $c_{n}<\alpha$.

(D) (convexity) $\int_{c_{L}}^{\alpha} \phi\left(\alpha, c_{n}, M, \sigma\right) \Gamma\left(c_{n}\right) d c_{n}<\left(\bar{c}-c_{L}\right) \phi\left(\alpha, c_{L}, M, \sigma\right)+(\alpha-\bar{c}) \phi(\alpha, \alpha, M, \sigma)$, where $\Gamma\left(c_{n}\right)=\frac{g^{\sigma}\left(c_{n}\right)+\left(c_{n}-\bar{c}\right) d g^{\sigma}\left(c_{n}\right) / d c_{n}}{g^{\sigma}\left(c_{L}\right)}$.

These assumptions are satisfied by the uniform distribution. Assumption 2.(B) is a monotonicity requirement. From Lemma (3), it also emerges that $\Phi(\alpha, M, \sigma)$ is decreasing in $\alpha$ and $\sigma$. Assumption 2.(C) is the single crossing condition: it guarantees the uniqueness of the equilibrium in the overall game. Assumption 2.(D) generally imposes a convexitylike condition, which is certainly verified if $\Gamma=1$ (as in the uniform case) and $\phi$ is convex in $c_{n}$.

Under Assumption 2.(B), if $\Phi\left(c_{H}, M, \sigma\right)<0$, then for every $M$, there exists an $\alpha^{*}$, such that: $E_{G_{\alpha^{*}}}\left[\tilde{\Pi}_{n} \mid c_{n}, M\right] \lessgtr 0$ when $c_{n} \gtrless \alpha^{*}$. Therefore, (30) implicitly defines the optimal entry threshold $\alpha^{*}$ :

$$
E_{G_{\alpha^{*}}}\left[\tilde{\Pi}_{n} \mid \alpha^{*}, M\right]=0
$$

Lemma 4 Assume that $\alpha^{*}$ is given by (30). Then, under Assumption 2:

$$
\frac{d \alpha^{*}}{d M}<0 ;\left.\quad \frac{\partial \alpha^{*}}{\partial D}\right|_{M}>0 ;\left.\quad \frac{\partial \alpha^{*}}{\partial \sigma}\right|_{M}<0 .
$$

When $\Phi\left(c_{H}, M, \sigma\right)>0, \alpha^{*}=c_{H}$ so that: $\frac{d \alpha^{*}}{d M}=0 ;\left.\frac{\partial \alpha^{*}}{\partial D}\right|_{M}=0$ and $\left.\frac{\partial \alpha^{*}}{\partial \sigma}\right|_{M}>0$. Note how the second inequality of (31) seems to suggest that an increase in the market density $D$ allows more inefficient firms to profitably stay in the market. However, this is not an equilibrium result for the full game because the partial derivatives in Lemma 4 are calculated for fixed $M$; hence they do not capture how a change in $D$ affects the equilibrium number of firms in the pre-entry stage.

\subsection{Pre-entry}

To determine $M$, we rely on the zero-profit condition. After paying $F_{e}$, firm $n$ enters the selection stage and learns $c_{n}$ and $M$. If $c_{n} \leq \alpha^{*}$, firm $n$ enters the market and gains 
$E_{G_{\alpha^{*}}}\left[\tilde{\Pi}_{n} \mid c_{n}, M\right]$, otherwise it stays out and gains 0 . The expected profit of the pre-entry stage, gross of the entry fee $F_{e}$, is:

$$
E\left[\tilde{\Pi}_{n} \mid M\right]=\int_{c_{L}}^{\alpha^{*}} E_{G_{\alpha^{*}}}\left[\tilde{\Pi}_{n} \mid c_{n}, M\right] d G\left(c_{n}\right)
$$

Lemma 5 Under Assumption 2, $E\left[\tilde{\Pi}_{n} \mid M\right]$ is monotonically decreasing in $M$.

The equilibrium number of entrants in the first stage, $M_{S}$ is implicitly given by:

$$
E\left[\tilde{\Pi}_{n} \mid M_{S}\right]=F_{e}
$$

and $N_{S}=M_{S} G\left(\alpha^{*}\left(M_{S}\right)\right)$. Under Lemma 5 the solution is unique. Comparative static results are summarized in the following propositions.

Proposition 8 Under Assumption 2,

$$
\frac{d \alpha^{*}}{d D}<0 ; \frac{d M_{S}}{d D}>0 ; \frac{d M_{S}}{d F_{e}}<0
$$

The first inequality mirrors the theoretical predictions in Syverson (2001, 2004): larger (denser) markets are characterized by higher levels of efficiency, and hence lower prices (Syverson, 2007). The second and third inequalities constitute a standard result: more potential entrants are attracted by denser markets and discouraged by higher entry fees. Market density has a direct and an undirect effect on the equilibrium number of firms, $N_{S}$ :

$$
\frac{d N_{S}}{d D}=\overbrace{\frac{d M_{S}}{d D}}^{>0} G\left(\alpha^{*}\right)+g\left(\alpha^{*}\right) \overbrace{\frac{d \alpha^{*}\left(M_{S}\right)}{d M}}^{<0} \overbrace{\frac{d M_{S}}{d D}}^{>0} M_{S} \gtreqless 0
$$

In a denser market, on the one hand, larger profits intensify entry in the first stage, thereby also increasing the number of firms in the production stage (direct effect); on the other, the competitive pressure from a larger number of firms push towards the exclusion of less competitive firms (indirect effect). The overall effect depends on the net balance between these two forces. Simulations obtained assuming a uniform distribution suggest that the first effect dominates: $N_{S}$ is increasing in $D$ but at a lower rate than $M_{S}$.

Proposition 9 Under Assumption 2:

$$
\frac{d M_{S}}{d \sigma}>0 ; \frac{d \alpha^{*}}{d \sigma} \lessgtr 0 \text { if } \Phi\left(c_{H}, M_{S}, \sigma\right) \lessgtr 0 .
$$


The first inequality confirms the result in Section 3, that cost heterogeneity increases expected profits and hence the number of potential entrants. However, the second inequality illustrates a counterbalancing effect: when selection is active, i.e. $\alpha^{*}<c_{H}$, the cut-off point is negatively related to costs' heterogeneity. The latter's impact on $N_{S}$ follows a pattern similar to (35), with the difference that in Figure 3, which plots $M_{S}, N_{S}$ and $\alpha^{*}$ as a function of $\left(c_{H}-c_{L}\right), N_{S}$ is decreasing. ${ }^{19}$ For low levels of $\left(c_{H}-c_{L}\right)$ (and therefore, variance), the optimal number of firms in the entry stage and in the production stage coincide $\left(M_{S}=N_{S}\right.$ and $\left.\alpha^{*}=c_{H}\right)$. However, as heterogeneity increases, the selection mechanism determines a cut-off point after which $\alpha^{*}<c_{H}$ and $M_{S}$ increases at a fast rate. As in the two-stage game, when the decision to enter is made under uncertainty, an increase in the costs' variance leads to an increase in the expected profit in the pre-entry stage, even when firms can anticipate that in the subsequent selection stage a number of firms will not continue. Most importantly, the selection mechanism is responsible for the decreasing trend in $N_{S}$ as it leads to the exit of the $M_{S}-N_{S}$ least efficient firms and to an increase of the average market efficiency, $\bar{c}_{e}=\frac{\alpha^{*}-c_{L}}{2}<\bar{c}$.

To further evaluate the effectiveness of the selection mechanism, we investigate the relationship between welfare and firms' heterogeneity. Figure 4 compares the expected total costs $E[C]$ in the three-stage selection game with those arising from three previously analyzed scenarios, all evaluated at the long-run equilibrium number of firms computed using (16), (25) and (33). ${ }^{20}$ The simulation shows that as firms' cost heterogeneity increases, the intensification of the selection mechanism produces such a gain in efficiency to push overall welfare towards levels close to those obtainable under uniform pricing regulation.

\section{Conclusions}

We have introduced firms' cost heterogeneity in the traditional model of the circular city (Salop, 1979), and shown that there exists an analytical solution for the equilibrium prices that holds for any number of firms and any distribution of costs that maintains localized competition as the relevant behavioral assumption. Our analysis suggests a very simple price setting rule: price is the weighted average of all firms' costs (plus the usual differentiation premium). The weights decrease quite sharply with distance, do not reflect any cost differential between any firms' pair and are largely independent of the number of firms.

\footnotetext{
${ }^{19}$ We have chosen the same parameters of Figure 2, assuming that $F=1$ is split into $F_{e}=0.2$ and $F_{p}=0.8$. The simulation further assumes $G$ is uniform, $\bar{c}=0.1, c_{h}-c_{l} \in[0,0.14], D=100$ and $t=1$.

${ }^{20}$ That is: $E[C]=\frac{1}{4} \hat{k}+\bar{c}+N F_{e} / D+M F_{p} / D-\sigma^{2} \hat{k}^{-1} \chi$ where $N=M=N_{F}$, with $\sigma=0, \chi=0$ under uniform pricing, $N=M=N_{M}, \chi \simeq 0.65$ under market pricing, $N=M=N_{F}, \chi=1$ under first-best pricing, and $N=N_{S}, M=M_{S}$ and $\chi \simeq 0.65$ under market pricing with selection.
} 
Introducing cost heterogeneity in location model opens up a number of interesting questions in terms of entry and price regulation, that we have only briefly explored. Two main conclusions seem to emerge from the long-run properties of two entry games. First, in the presence of large cost heterogeneity, a regulatory approach combining uniform pricing and entry restrictions is allocatively inefficient relative to the sole regulation on the number of market participants.

Second, when firms have to pay two entry sunk fees, the first to learn their own cost realization, the other to enter the production stage, the free market equilibrium endogenously determines a selection process that generates significant (and increasing) welfare effects starting from intermediate levels of firms' heterogeneity. Entry into such liberal professions as accountants, engineers, lawyers, pharmacists, and notaries, fits this theoretical framework quite well; the first entry cost being the cost of acquiring professional accreditation, the second corresponding to the setting-up of the practice or shop. ${ }^{21}$ According to our results, liberalization is likely to be a welfare-enhancing strategy in those markets characterized by a certain degree of ex-ante variability in firms' costs. Our theoretical analysis thus supports the stance recently taken by the European Commission to commit to facilitate the introduction of pro-competitive mechanisms into the liberal professions' markets - see the Commission Communication COM (2004) 83. A more in depth investigation of these, and other issues, is left to future research.

\section{Appendix}

Proof of Lemma 1. Express the system of equations (2) in matrix form:

$$
A=\left(\begin{array}{ccccccccc}
1 & -\frac{1}{4} & 0 & . . & . . & . . & . . & 0 & -\frac{1}{4} \\
-\frac{1}{4} & 1 & -\frac{1}{4} & 0 & . . & . . & . . & . . & 0 \\
0 & -\frac{1}{4} & 1 & -\frac{1}{4} & 0 & . . & . . & . . & 0 \\
. . & . . & . . & . . & . . & . . & & & . . \\
. . & & & & . . & . . & . . & . . & 0 \\
0 & . . & . . & . . & . . & 0 & -\frac{1}{4} & 1 & -\frac{1}{4} \\
-\frac{1}{4} & 0 & . . & . . & . . & . . & 0 & -\frac{1}{4} & 1
\end{array}\right) ; P=\left(\begin{array}{c}
p_{\underline{l}} \\
p_{\underline{l}+1} \\
. . \\
p_{0} \\
. . \\
p_{\bar{l}-1} \\
p_{\bar{l}}
\end{array}\right) ; C=\left(\begin{array}{c}
c_{\underline{l}} \\
c_{\underline{l}+1} \\
. . \\
c_{0} \\
. . \\
c_{\underline{l}+1} \\
c_{\bar{l}-1}
\end{array}\right)
$$

\footnotetext{
${ }^{21}$ The cost to acquire a university degree is generally not sunk, as the degree can be used to pursue a number of different career options.
} 
Since $A$ is strictly diagonal dominant by rows (i.e. $\left|a_{i i}\right|>\sum_{j \neq i}\left|a_{i j}\right|$ ), it is non singular and hence the solution of the system is unique (Bhatia, 1997, p. 251). Linearity of (2) also implies that the solution is linear in $c_{n}$, i.e. $p_{n}=\sum_{i=\underline{l}}^{\bar{l}} w_{i, n} c_{\langle n+i\rangle}+k_{n}$. In addition, the structure of $A$ also implies that: a) $w_{i, n}=w_{i}$ and $k_{n}=k$, for $n \in \mathbb{L}$, as every $i$-step shift of the position of all firms on the circle must not affect the result; b) $w_{i}=w_{-i}$, as mirroring the position of the firms on the circle, the result must remain unchanged.

\section{Proof of Proposition 1.}

Under Condition 1, the best reply function of firm $n$ is given by (2), for $n \in \mathbb{L}$. From Lemma (1), the solution of the system is unique, and must be of the form given in (3). We show that if firm $n$ 's adjacent rivals located at $\langle n-1\rangle$ and $\langle n+1\rangle$ choose $p_{\langle n-1\rangle}^{*}$ and $p_{\langle n+1\rangle}^{*}$, where the weights $w_{i}$ and $k$ are given by (4) and (5), respectively, then for firm $n$ it is optimal to set:

$$
p_{n}^{*}=\sum_{i=\underline{l}}^{\bar{l}} w_{\bar{l}-|i|} c_{\langle n+i\rangle}+k,
$$

with weights given by (4) and (5). Replacing $p_{\langle n-1\rangle}$ and $p_{\langle n+1\rangle}$ in (2), we obtain:

$$
p_{n}=\frac{1}{4}\left[2 c_{n}+\left(c_{\langle n+i\rangle}+\sum_{i=\underline{l}}^{\bar{l}} w_{\bar{l}-|i|} \cdot c_{\langle n-1+i\rangle}+k\right)+\left(\sum_{i=\underline{l}}^{\bar{l}} w_{\bar{l}-|i|} \cdot c_{\langle n+1+i\rangle}+k\right)\right]+\frac{1}{2} \hat{k}
$$

We derive the proofs for $N$ even and odd, separately.

Even case: $N=2$ is trivial. Consider $N \geq 4$. Collect the similar terms for $c_{\langle n+i\rangle}, i \in \mathbb{L}$ :

$$
\begin{aligned}
p_{n}= & \frac{1}{4}\left[\sum_{i=\underline{\underline{l}}}^{-1}\left(w_{\bar{l}-|i|-1}+w_{\bar{l}-|i|+1}\right) \cdot c_{\langle n+i\rangle}+2\left(1+w_{\bar{l}-1}\right) c_{n}+\right. \\
& \left.+\sum_{i=+1}^{\bar{l}-1}\left(w_{\bar{l}-|i|-1}+w_{\bar{l}-|i|+1}\right) \cdot c_{\langle n+i\rangle}+2 w_{1} \cdot c_{\langle n+\bar{l}\rangle}\right]+\frac{1}{2}(\hat{k}+k)
\end{aligned}
$$

Requiring all coefficients of $c_{\langle n+i\rangle}$ to be the same in (37) and (38), we obtain (4a)-(4c). A similar reasoning can be used to derive (5). Finally, it is easy to show that (4d) is satisfied, by replacing $c_{n}=c$ and $k=\hat{k}$ in (37) and (38), and equating the two expressions.

Odd case: The proof is very similar to the even case. Collecting the similar terms for $c_{n}$ :

$$
\begin{aligned}
p_{n}= & \frac{1}{4}\left[\left(w_{0}+w_{1}\right) c_{n+\underline{l}}+\sum_{i=\underline{l}+1}^{-1}\left(w_{\bar{l}-|i|-1}+w_{\bar{l}-|i|+1}\right) \cdot c_{n+i}+2\left(1+w_{\bar{l}-1}\right) c_{n}+\right. \\
& \left.+\sum_{i=+1}^{\bar{l}-1}\left(w_{\bar{l}-|i|-1}+w_{\bar{l}-|i|+1}\right) \cdot c_{n+i}+2\left(w_{0}+w_{1}\right) \cdot c_{\langle n+\bar{l}\rangle}\right]+\frac{1}{2}(\hat{k}+k) .
\end{aligned}
$$

The only difference with the even case is the second equality of system (4). 
Proof of Corollary 1. We show that $w_{i}$ described in (4) can be generated from system (10) and (11). Equations (4a), (4b) and (4d) are sufficient to uniquely determine $w_{i}$ (thus $4 \mathrm{c}$ is unnecessary). Let $\hat{w}_{i}=a(i) / A_{N}$. Rewriting (10a), (10b) and (10c) in terms of $\hat{w}_{i}$ yields, respectively, (4a), (4b) and (4d), woth $w_{i}$ replaced by $\hat{w}_{i}$. Thus: $\hat{w}_{i}=w_{i}$.

Proof of Corollary 2. Because (10a) and (10b) describe a linear recurrence of order two, with constant coefficients, hence it has a unique closed-form solution. Standard techniques imply that $a(j)=\frac{1}{2}(2+\sqrt{3})^{j}+\frac{1}{2}(2-\sqrt{3})^{j}$ in the even case; and $a(j)=\left(\frac{1}{6} \sqrt{3}+\frac{1}{2}\right)(2+\sqrt{3})^{j}+$ $\left(\frac{1}{2}-\frac{1}{6} \sqrt{3}\right)(2-\sqrt{3})^{j}$ in the odd case. Taking $\bar{l} \rightarrow \infty$ then $w_{\bar{l}} / w_{\bar{l}-1}=a(\bar{l}) / a(\bar{l}-1) \rightarrow x=$ $(2+\sqrt{3})$, and more generally $w_{\bar{l}} / w_{\bar{l}-i} \rightarrow x^{i}$.

Note that $\frac{1}{w_{\bar{l}}}=\frac{A(\bar{l})}{a(\bar{l})}=\frac{a(\bar{l})}{a(\bar{l})}+2 \frac{a(\bar{l}-1)}{a(\bar{l})}+2 \frac{a(\bar{l}-2)}{a(\bar{l})}+. .+(3-\underline{l}-\bar{l}) \frac{a(0)}{a(\bar{l})}$. For $\bar{l}$ large, $\frac{1}{w_{\bar{l}}} \simeq$ $1+2 \sum_{i=1}^{\bar{l}-1} \frac{1}{x^{i}}$. Taking $\bar{l} \rightarrow \infty$ then $\frac{1}{w_{\bar{l}}} \rightarrow 1+(\sqrt{3}-1)=\sqrt{3}$.

\section{Proof of Proposition 2.}

(Existence) We start by computing the maximal costs' differentials $c_{H}-c_{L}$ for which Condition (1) is satisfied, i.e.: $-\hat{k}<p_{n}^{*}-p_{\langle n-1\rangle}^{*}<\hat{k}$. Without loss of generality, assume that $p_{n}^{*}>p_{\langle n-1\rangle}^{*}$. Using (3)-(5) yields:

$$
\begin{aligned}
p_{n}^{*}-p_{\langle n-1\rangle}^{*}= & {\left[\left(w_{0}-w_{\bar{l}+\underline{l}}\right) c_{\langle n+\bar{l}\rangle}+\sum_{i=-\bar{l}}^{-1}\left(w_{\bar{l}-|i|}-w_{\bar{l}-|i+1|}\right) c_{\langle n+i\rangle}\right] } \\
& +\left[\sum_{i=0}^{\bar{l}-1}\left(w_{\bar{l}-|i|}-w_{\bar{l}-|i+1|}\right) c_{\langle n+i\rangle}\right] .
\end{aligned}
$$

Because all the terms multiplying the $c_{\zeta .\rangle}$ in the first square bracket are negative, and all those in the second bracket are positive, the maximal price difference for a given cost differential is given by choosing $c_{\langle n+i\rangle}=c_{L}$, for $i=\underline{l}, . .,-1, \bar{l}$, and $c_{\langle n+i\rangle}=c_{H}$ for $i=0, . ., \bar{l}-1$. Let:

$$
\rho_{c}=\left[-\left(w_{0}-w_{\bar{l}+\underline{l}}\right)-\sum_{i=\underline{l}}^{-1}\left(w_{\bar{l}-|i|}-w_{\bar{l}-|i+1|}\right)\right]^{-1}=\left[\sum_{i=0}^{\bar{l}-1}\left(w_{\bar{l}-|i|}-w_{\bar{l}-|i+1|}\right)\right]^{-1}>1 \text {. Con- }
$$
dition (1) is satisfied by (12).

(Uniqueness) It is given by Lemma 1.

\section{Proof of Proposition 3.}

(Existence) We focus on the situation in which costs differentials are such that: $c_{H}-c_{L}<\hat{k}$. From (12), $p^{*}=\left(p_{\underline{l}}^{*}, . . p_{\bar{l}}^{*}\right)$ given by (3)-(5) satisfies Condition (1). In order to be an equilibrium, $p^{*}$ must be such that, a generic firm $n$ has no unilateral incentive to deviate from $p_{n}^{*}$. Assume that $\hat{p}_{n}$ is a unilateral deviation potentially profitable for firm $n$. Since Condition 1 ensures the uniqueness of the solution, we require that $\hat{p}_{n}$ is such that it does not satisfy this Condition.

If $\hat{p}_{n}>\min \left\{p_{\langle n-1\rangle}^{*}, p_{\langle n+1\rangle}^{*}\right\}+\hat{k}$ then firm $n$ is undercut by at least one of the opponents, so 
that its profit is null: therefore this strategy is not profitable.

But if firm $n$ chooses $\hat{p}_{n}<\max \left\{p_{\langle n-1\rangle}^{*}, p_{\langle n+1\rangle}^{*}\right\}-\hat{k}$, it can grab the market of at least one adjacent opponent. Clearly, the maximum advantage from deviation emerges when $p_{\langle n-1\rangle}^{*}$ and $p_{\langle n+1\rangle}^{*}$ are as large as possible, so that after undercutting a firm retains the largest margin. Equations (3)-(5) imply that $c_{L}<p_{n}^{*}-\hat{k}<c_{H}, n \in \mathbb{L}$. Hence, for a firm with costs sufficiently low relative to the 1-step rivals, it is possible to choose $\hat{p}_{n}>c_{n}$ such that $\hat{p}_{n} \leq p_{\langle n+1\rangle}^{*}-\hat{k}$ and $\hat{p}_{n} \leq p_{\langle n-1\rangle}^{*}-\hat{k}$. But if we assume that $c_{H}-c_{L}<\hat{k}$, it is not possible that $\hat{p}_{n}>c_{n}$ and simultaneously $\hat{p}_{n} \leq p_{\langle n-2\rangle}^{*}-2 \hat{k}$ or $\hat{p}_{n} \leq p_{\langle n+2\rangle}^{*}-2 \hat{k}$. So firm $n$ can profitable undercut one or both its 1-step opponents but not any $i$-step rival, with $i>1$.

Assume the best possible situation for firm $n$, i.e., $c_{n}=c_{L}$ and $c_{\langle n+i\rangle}=c_{H}$ for $i \in \mathbb{L}_{0} \equiv \mathbb{L} \backslash\{0\}$. For symmetry $p_{\langle n+i\rangle}^{*}=p_{\langle n-i\rangle}^{*}$ for $i \in \mathbb{L}_{0}$. Further, by choosing $p_{n}=p_{n}^{*}$, firm $n$ obtains the profit of: $\Pi_{n}^{*}=\frac{D}{t}\left(p_{n}^{*}-c_{n}\right)\left(p_{\langle n-1\rangle}^{*}-p_{n}^{*}+k\right)$.

If firm $n$ undercuts its rivals, it must choose a price $\hat{p}_{n} \leq p_{\langle n-1\rangle}^{*}-k$, so that its next competitors become $\langle n-2\rangle$ and $\langle n+2\rangle$. For analytical convenience, we assume that by choosing $\hat{p}_{n}=p_{\langle n-1\rangle}^{*}-$ $\hat{k}$, firm $n$ is able to "steal" all the customers of firm $\langle n-1\rangle$ and sells to $\frac{D}{t}\left(p_{\langle n-2\rangle}^{*}-\hat{p}_{n}+2 \hat{k}\right)$ consumers; consequently, the profit it gains is: $\frac{D}{t}\left(\hat{p}_{n}-c_{n}\right)\left(p_{\langle n-2\rangle}^{*}-\hat{p}_{n}+2 k\right)$. Since the previous expression, which is concave in $\hat{p}_{n}$, is maximized in $\frac{1}{2} p_{\langle n-2\rangle}^{*}+\frac{1}{2} c_{n}+\hat{k}>p_{\langle n-1\rangle}^{*}-\hat{k}$, the maximum deviation profit $\Pi_{n}^{d}$ (satifying $\hat{p}_{n} \leq p_{\langle n-1\rangle}^{*}-\hat{k}$ ) is reached when $\hat{p}_{n}=p_{\langle n-1\rangle}^{*}-\hat{k}$ with $\Pi_{n}^{d}=$ $\frac{D}{t}\left(\left(p_{\langle n-1\rangle}^{*}-\hat{k}\right)-c_{n}\right)\left(p_{\langle n-2\rangle}^{*}-\left(p_{\langle n-1\rangle}^{*}-\hat{k}\right)+2 \hat{k}\right)$. In order for deviation to be unprofitable, $\Pi_{n}^{*}-\Pi_{n}^{d}>0$. Divide the latter by $D / k t$ and replace $p_{n}^{*}, p_{\langle n-1\rangle}^{*}$ and $p_{\langle n-2\rangle}^{*}$, with the respective analitical expressions provided by (3)-(5), and set $\gamma=\left(c_{H}-c_{L}\right) / \hat{k}$ to obtain:

$$
\left(\left(1-w_{\bar{l}}\right) \gamma+1\right)\left(\left(w_{\bar{l}}-w_{\bar{l}-1}\right) \gamma+1\right)-\left(1-w_{\bar{l}-1}\right)\left(\left(w_{\bar{l}-1}-w_{\bar{l}-2}\right) \gamma+3\right) \gamma>0 .
$$

After some algebra, it emerges that this is satisfied if:

$$
\left(c_{H}-c_{L}\right)<\rho(N) \hat{k}
$$

where $\rho(N)=\frac{1}{A}\left(B-\sqrt{B^{2}-A}\right)$ and $A=\left(1-w_{\bar{l}-1}\right)^{2}-\left(1-w_{\bar{l}}\right)^{2}-\left(1-w_{\bar{l}-1}\right)\left(w_{\bar{l}}-w_{\bar{l}-2}\right)$ and $B=\left(1-w_{\bar{l}-1}\right)$. When $N=2$ firms have no incentive to undercut the rivals, so that $\rho(2)=\rho_{c}(2)=3$.

(Uniqueness) We show that every profile strategy $\hat{p} \neq p^{*}$ cannot be an equilibrium. Assume that another price strategy equilibrium vector $\hat{p} \neq p^{*}$ exists. It means that for some $n$, Condition 1 is not satisfied, i.e. there exists at least one $\hat{p}_{n}$ such that $\left|\hat{p}_{n}-\hat{p}_{\langle n+1\rangle}\right|>\hat{k}$. Let $\mathbb{L}_{n p}$ be the set 
of firms that in equilibrium do not sell to any consumer. Note that for any $\hat{p}$, at least one but not all firms must sell a positive quantity, i.e. $1 \leq \# \mathbb{L}_{n p} \leq N-1$. In equilibrium, a firm cannot have negative profits, otherwise it would prefer to charge a sufficiently high price and sell nothing. Let $n \in \mathbb{L}_{n p}$. Because $n$ sells nothing, then at least one of the following conditions must hold: $\hat{p}_{n}>\hat{p}_{\langle n+i\rangle}+|i| \hat{k}$, for $0 \neq i \in \mathbb{L}$. Now, choose $\varepsilon>0$ sufficiently small. If $n$ charges $\check{p}_{n}=c_{n}+\varepsilon$, it can profitably deviate and reach a positive profit, (otherwise at least one active opponent is gaining negative profits) Hence, assuming the existence of another equilibrium $\hat{p} \neq p^{*}$ is a contradiction.

Proof of Proposition 4. Profit (14) can be decomposed as:

$$
\begin{aligned}
& E\left[\tilde{\Pi}_{n}\right]=\frac{D t}{N^{2}}-F+ \\
& \frac{D}{2 t}\left\{E\left[\tilde{p}_{n} \tilde{p}_{\langle n-1\rangle}\right]-2 E\left[\tilde{p}_{n}^{2}\right]+2 E\left[\tilde{c}_{n} \tilde{p}_{n}\right]-E\left[\tilde{c}_{n} \tilde{p}_{\langle n-1\rangle}\right]-E\left[\tilde{c}_{n} \tilde{p}_{\langle n+1\rangle}\right]+E\left[c_{n} \tilde{p}_{\langle n+1\rangle}\right]\right\} .
\end{aligned}
$$

The expected values in (39) are derived in different parts.

Part 4.I Under Assumption 1, if firms set their prices following Proposition (1), then $E\left[\left(\tilde{p}_{n}\right)^{2}\right]=\sigma^{2} W_{I .0}(N)+\bar{c}^{2}+2 \bar{c} \hat{k}+\hat{k}^{2}$ where $W_{I .0}(N)=\sum_{i=\underline{l}}^{\bar{l}} w_{\bar{l}-|i|}^{2}>0$.

Derivation of Part 4.I. From (3): $E\left[\left(\tilde{p}_{n}\right)^{2}\right]=E\left[\left(\sum_{i=\underline{l}}^{\bar{l}} w_{\bar{l}-|i|} \cdot \tilde{c}_{\langle n+i\rangle}+\hat{k}\right)^{2}\right]$ which can be decomposed in three terms: $E\left[\left(\sum_{i=\underline{l}}^{\bar{l}} w_{\bar{l}-|i|} \cdot \tilde{c}_{\langle n+i\rangle}\right)^{2}\right]+2 E\left[\left(\sum_{i=\underline{l}}^{\bar{l}} w_{\bar{l}-|i|} \cdot \tilde{c}_{\langle n+i\rangle}\right)\right] \hat{k}+\hat{k}^{2}$. Under Assumption 1, we know that $E\left[\tilde{c}_{n}^{2}\right]=\left(\sigma^{2}+\bar{c}^{2}\right)$, and, since $\tilde{c}_{n}$ and $\tilde{c}_{\langle n+i\rangle}$ are uncorrelated: $E\left[\tilde{c}_{n} \tilde{c}_{\langle n+i\rangle}\right]=\bar{c}^{2}$, with $i \in \mathbb{L}_{0}$. After rewriting $E\left[\left(\sum_{i=\underline{l}}^{\bar{l}} w_{\bar{l}-|i|} \cdot \tilde{c}_{\langle n+i\rangle}\right)^{2}\right]$ as:

$$
\sum_{i=\underline{l}}^{\bar{l}} \sum_{j=\underline{l}}^{\bar{l}} w_{\bar{l}-|i|} w_{\bar{l}-|j|} E\left[\tilde{c}_{\langle n+i\rangle} \cdot \tilde{c}_{\langle n+j\rangle}\right]
$$

only two types of terms are left: $E\left[\tilde{c}_{n}^{2}\right]$ and $E\left[\tilde{c}_{n} \tilde{c}_{\langle n+i\rangle}\right], i \in \mathbb{L}_{0}$. Further, $\sum_{i=\underline{l}}^{\bar{l}} \sum_{j=\underline{l}}^{\bar{l}} w_{\bar{l}-|i|} w_{\bar{l}-|j|}=$ 1. Since the total weight involving $E\left[\tilde{c}_{n}^{2}\right]$ terms is $W_{I .0}(N)=\sum_{i=\underline{l}}^{\bar{l}} w_{\bar{l}-|i|}^{2}$, we immediately obtain the thesis. $\diamond$

Part 4.II Under Assumption 1, if firms set their prices following Proposition 1, then $E\left[\tilde{p}_{n} \tilde{p}_{n-1}\right]=\sigma^{2} W_{I .1}(N)+\bar{c}^{2}+2 \bar{c} \hat{k}+\hat{k}^{2}$ where $W_{I .1}(N)=\sum_{i=\underline{l}}^{\bar{l}} w_{\bar{l}-|i|} w_{\bar{i}-|i+1|}>0$.

Derivation of Part 4.II. From (3):

$$
E\left[\tilde{p}_{n} \tilde{p}_{n-1}\right]=E\left[\left(\sum_{i=\underline{l}}^{\bar{l}} w_{\bar{l}-|i|} \cdot \tilde{c}_{\langle n+i\rangle}+\hat{k}\right)\left(\sum_{i=\underline{l}}^{\bar{l}} w_{\bar{l}-|i|} \cdot \tilde{c}_{\langle n+i-1\rangle}+\hat{k}\right)\right]
$$


The decomposition of the expected value is similar to that presented in the proof of Part (4.I). To obtain the proof of the proposition, note that the total weight involving $E\left[\tilde{c}_{n}^{2}\right]$ terms is $W_{I .1}(N)=$ $\sum_{i=\underline{l}}^{\bar{l}} w_{\bar{l}-|i|} w_{\bar{l}-|i+1|} \cdot \diamond$

Part 4.III Under Assumption 1, if firms set their prices following Proposition 1, then:

a) $E\left[\tilde{c}_{n} \tilde{p}_{n}\right]=\sigma^{2} w_{\bar{l}}+\bar{c}^{2}+\bar{c} \hat{k}$; and b) $E\left[\tilde{c}_{n} \tilde{p}_{\langle n-1\rangle}\right]=\sigma^{2} w_{\bar{l}-1}+\bar{c}^{2}+\bar{c} \hat{k}$.

Derivation of Part 4.III. a) $E\left[\tilde{c}_{n} \tilde{p}_{n}\right]=E\left[\tilde{c}_{n}\left(\sum_{i=\underline{l}}^{\bar{l}} w_{\bar{l}-|i|} \cdot \tilde{c}_{n+i}+\hat{k}\right)\right]$. The thesis follows from the same reasonings of Parts (4.I) and (4.II) by noting that the weight associated to $E\left[\tilde{c}_{n}^{2}\right]$ is $w_{\bar{l}}$.

b). Similar to a). In this case the weight of $E\left[\tilde{c}_{n}^{2}\right]$ is $w_{\bar{l}-1} \cdot \diamond$

To complete the proof of the Proposition, consider that firms lack information on their own and their opponents' costs and locations; therefore $E\left[\tilde{p}_{n} \tilde{p}_{\langle n+1\rangle}\right]=E\left[\tilde{p}_{n} \tilde{p}_{\langle n-1\rangle}\right]$ and $E\left[c_{n} \tilde{p}_{\langle n+1\rangle}\right]=$ $E\left[c_{n} \tilde{p}_{\langle n-1\rangle}\right]$. After substituting the results from Parts 4.I, 4.II, and 4.III into (39), most of the terms cancel out, finally yielding: $E\left[\tilde{\Pi}_{n}\right]=\frac{D}{t}\left(\hat{k}^{2}+\sigma^{2} W_{I}(N)\right)-F$, where $W_{I}(N):=$ $W_{I .0}-W_{I .1}+w_{\bar{l}}-w_{\bar{l}-1}=\sum_{i=\underline{l}}^{\bar{l}} w_{\bar{l}-|i|}\left(\left(w_{\bar{l}}-w_{\bar{l}-|i|}\right)-\left(w_{\bar{l}-1}-w_{\bar{l}-|i+1|}\right)\right)$. To compute $\bar{W}_{I}$, note that $W_{I}(N)=\left(w_{\bar{l}}-w_{\bar{l}-1}\right)-\sum_{i=\underline{l}}^{\bar{l}} w_{\bar{l}-|i|} \cdot\left(w_{\bar{l}-|i|}-w_{\bar{l}-|i+1|}\right)$. Using Lemma 1, and choosing $z=x^{-1}, W_{I}(N)$, for $N$ large enough $W_{I}(N) \simeq(y-z y)+\sum_{i=\underline{l}}^{\bar{l}}\left(y z^{|i|} \cdot y z^{|i+1|}\right)-\sum_{i=\underline{l}}^{\bar{i}}\left(y z^{|i|}\right)^{2}=$ $(y-z y)+y^{2} \frac{z-1}{z+1}=1-\frac{4}{9} \sqrt{3} \simeq 0.23020$.

Proof of Proposition 5. It derives directly from Proposition 4.

Proof of Proposition 7. Multiply the terms within the square brackets in (26) to obtain: $E\left[\tilde{p}_{n} \tilde{p}_{\langle n-1\rangle}\right]-E\left[\tilde{p}_{n}^{2}\right]+E\left[\tilde{p}_{n}\right] \hat{k}-c_{n} E\left[\tilde{p}_{\langle n-1\rangle}\right]+c_{n} E\left[\tilde{p}_{n}\right]-c_{n} \hat{k}$. Each term is derived separately.

Part 7.I Under Assumption 1, if firm $n$ knows its costs but not those of its opponents and in the final stage prices follow Proposition 1, then

$E_{G_{e}}\left[\tilde{p}_{\langle n\rangle}^{2}\right]=\left(\left(w_{\bar{l}} c_{n}+\left(1-w_{\bar{l}}\right) \bar{c}_{e}\right)+\hat{k}\right)^{2}+\sigma_{e}^{2} W_{I I .0}(N)$, where $W_{I I .0}(N)=\sum_{i=\mathbb{L}_{0}}\left(w_{\bar{l}-|i|}\right)^{2}$.

Derivation of Part 7.I. To save notation, we do not explicitly show the dependence of the expected value on $G_{e}$. Using (3), $E\left[c_{n}\right]=c_{n}, E\left[\tilde{c}_{\langle n+i\rangle}\right]=\bar{c}_{e}, E\left[c_{n}^{2}\right]=c_{n}^{2}, E\left[c_{n} \tilde{c}_{\langle n+i\rangle}\right]=c_{n} \bar{c}_{e}$, $E\left[\tilde{c}_{\langle n+i\rangle}^{2}\right]=\left(\sigma^{2}+\bar{c}_{e}^{2}\right)$, with $i \in \mathbb{L}_{0}$ and $E\left[\tilde{c}_{\langle n+i\rangle} \tilde{c}_{\langle n+j\rangle}\right]=\bar{c}^{2}, i, j \in \mathbb{L}_{0}, i \neq j$ :

$$
\begin{aligned}
E\left[\tilde{p}_{\langle n\rangle}^{2}\right]= & E\left[\left(w_{\bar{l}} \cdot c_{n}+\sum_{i=\mathbb{L}_{0}} w_{\bar{l}-|i|} \cdot \tilde{c}_{\langle n+i\rangle}+\hat{k}\right)^{2}\right] \\
& E\left[\left(w_{\bar{l}} \cdot c_{n}+\sum_{i=\mathbb{L}_{0}} w_{\bar{l}-|i|} \cdot \tilde{c}_{\langle n+i\rangle}\right)^{2}\right]+2\left(w_{\bar{l}} \cdot c_{n}+\left(1-w_{\bar{l}}\right) \bar{c}_{e}\right) \hat{k}+\hat{k}^{2} .
\end{aligned}
$$


The expected value in the squared brackets can be written as: $w_{\bar{l}}^{2} c_{n}^{2}+2 w_{\bar{l}} \sum_{i=\mathbb{L}_{0}} w_{\bar{l}-|i|} \bar{c}_{e} c_{n}+$ $\sum_{i=\mathbb{L}_{0}} w_{\bar{l}-|i|}^{2} E\left[\tilde{c}_{\langle n+i\rangle}^{2}\right]+\sum_{i \neq j ; i, j=\mathbb{L}_{0}} w_{\bar{l}-|j|} w_{\bar{l}-|i|} E\left[\tilde{c}_{\langle n+i\rangle} \tilde{c}_{\langle n+j\rangle}\right]$, or:

$$
w_{\bar{l}}^{2} c_{n}^{2}+2 w_{\bar{l}}\left(1-w_{\bar{l}}\right) \bar{c}_{e} c_{n}+\sum_{i=\mathbb{L}_{0}} w_{\bar{l}-|i|}^{2} \sigma_{e}^{2}+\sum_{i, j=\mathbb{L}_{0}} w_{\bar{l}-|j|} w_{\bar{l}-|i|} \bar{c}_{e}^{2}
$$

Setting $W_{I I .0}(N)=\sum_{i=\mathbb{L}_{0}}\left(w_{\bar{l}-|i|}\right)^{2}$ and noting that $\sum_{i, j=\mathbb{L}_{0}} w_{\bar{l}-|j|} w_{\bar{l}-|i|}=\left(1-w_{\bar{l}}\right)^{2}$, we obtain $\left(w_{\bar{l}} c_{n}+\left(1-w_{\bar{l}}\right) \bar{c}_{e}\right)^{2}+\sigma_{e}^{2} W_{I I .0}(N)$. Summing up previous results completes the calculation. $\diamond$

Part 7.II Under Assumption 1, if firm $n$ knows its costs but not those of its opponent and in the final stage prices follow Proposition 1, then

$E_{G_{e}}\left[\tilde{p}_{\langle n\rangle} \tilde{p}_{\langle n-1\rangle}\right]=\left(c_{n} w_{\bar{l}-1}+\left(1-w_{\bar{l}-1}\right) c_{e}+\hat{k}\right)\left(c_{n} w_{\bar{l}}+\left(1-w_{\bar{l}}\right) c_{e}+\hat{k}\right)+W_{I I .1}(N) \sigma^{2}$, where $W_{I I .1}(N)=\sum_{i=\mathbb{L}_{0}} w_{\bar{l}-|i|} w_{\bar{l}-|i+1|}$.

Derivation of Part 7.II. The proof is similar to that of Part 7.I. Using (3), we obtain: $E\left[\tilde{p}_{\langle n\rangle} \tilde{p}_{\langle n-1\rangle}\right]=E\left[\left(w_{\bar{l}} \cdot c_{n}+\sum_{i=\mathbb{L}_{0}} w_{\bar{l}-|i|} \cdot \tilde{c}_{\langle n+i\rangle}+\hat{k}\right)\left(w_{\bar{l}-1} \cdot c_{n}+\sum_{i=\mathbb{L}_{0}} w_{\bar{l}-|i+1|} \cdot \tilde{c}_{\langle n+i\rangle}+\hat{k}\right)\right]$.

This can be written as:

$$
\begin{gathered}
E\left[\left(w_{\bar{l}} c_{n}+\sum_{i=\mathbb{L}_{0}} w_{\bar{l}-|i|} \tilde{c}_{\langle n+i\rangle}\right)\left(w_{\bar{l}-1} c_{n}+\sum_{i=\mathbb{L}_{0}} w_{\bar{l}-|i+1|} \tilde{c}_{\langle n+i\rangle}\right)\right]+ \\
+\left(w_{\bar{l}} c_{n}+\sum_{i=\mathbb{L}_{0}} w_{\bar{l}-|i|} \bar{c}_{e}\right) \hat{k}+\left(w_{\bar{l}-1} c_{n}+\sum_{i=\mathbb{L}_{0}} w_{\bar{l}-|i+1|} \tilde{c}_{\langle n+i\rangle}\right) \hat{k}+\hat{k}^{2} . \text { Or: } \\
w_{\bar{l}} w_{\bar{l}-1} c_{n}^{2}+w_{\bar{l}}\left(1-w_{\bar{l}-1}\right) c_{n} \bar{c}_{e}+w_{\bar{l}-1}\left(1-w_{\bar{l}}\right) c_{n} \bar{c}_{e}+\sum_{i=\mathbb{L}_{0}} w_{\bar{l}-|i|} w_{\bar{l}-|i+1|}\left(\sigma^{2}+\bar{c}_{e}^{2}\right) \\
+\sum_{i \neq j ; i, j=\mathbb{L}_{0}} w_{\bar{l}-|i|} w_{\bar{l}-|j+1|} \bar{c}_{e}^{2}+\left(w_{\bar{l}} \cdot c_{n}+\left(1-w_{\bar{l}}\right) \bar{c}_{e}\right) k+\left(w_{\bar{l}-1} c_{n}+\left(1-w_{\bar{l}-1}\right) \bar{c}_{e}\right) \hat{k}+\hat{k}^{2} .
\end{gathered}
$$

Noting that $\sum_{i, j=\mathbb{L}_{0}} w_{\bar{l}-|i|} w_{\bar{l}-|j+1|}=\left(1-w_{\bar{l}}\right)\left(1-w_{\bar{l}-1}\right)$, after some simplifications:

$$
\begin{aligned}
& w_{\bar{l}} w_{\bar{l}-1} c_{n}^{2}+w_{\bar{l}}\left(1-w_{\bar{l}-1}\right) c_{n} \bar{c}_{e}+w_{\bar{l}-1}\left(1-w_{\bar{l}}\right) c_{n} \bar{c}_{e}+\left(1-w_{\bar{l}}\right)\left(1-w_{\bar{l}-1}\right) \bar{c}_{e}^{2}+W_{I I .1}(N) \sigma^{2}+ \\
& +\left(w_{\bar{l}} \cdot c_{n}+\left(1-w_{\bar{l}}\right) \bar{c}_{e}\right) \hat{k}+\left(w_{\bar{l}-1} c_{n}+\left(1-w_{\bar{l}-1}\right) \bar{c}_{e}\right) \hat{k}+\hat{k}^{2},
\end{aligned}
$$

with: $W_{I I .1}(N)=\sum_{i=\mathbb{L}_{0}} w_{\bar{l}-|i|} w_{\bar{l}-|i+1|}$. The thesis directly follows from factorization. $\diamond$

a) To derive (27), substitute $E\left[\tilde{p}_{n}\right]=w_{\bar{l}} c_{n}+\left(1-w_{\bar{l}}\right) \bar{c}_{e}+\hat{k}, E\left[\tilde{p}_{\langle n-1\rangle}\right]=w_{\bar{l}-1} c_{n}+\left(1-w_{\bar{l}-1}\right) \bar{c}_{e}$ 
$+\hat{k}$ and the results in Parts 7.I and 7.II into (26):

$$
\begin{aligned}
& E_{G_{e}}\left[\tilde{\Pi}_{n} \mid c_{n}, N, M\right]= \\
& \left(c_{n} w_{\bar{l}-1}+\left(1-w_{\bar{l}-1}\right) \bar{c}_{e}+\hat{k}\right)\left(c_{n} w_{\bar{l}}+\left(1-w_{\bar{l}}\right) \bar{c}_{e}+\hat{k}\right)+W_{I I .1} \sigma^{2} \\
& -\left(\left(w_{\bar{l}} c_{n}+\left(1-w_{\bar{l}}\right) \bar{c}_{e}\right)+\hat{k}\right)^{2}-\sigma_{e}^{2} W_{I I .0}+\left(w_{\bar{l}} c_{n}+\left(1-w_{\bar{l}}\right) \bar{c}_{e}+\hat{k}-c_{n}\right) \hat{k} \\
& -c_{n}\left(w_{\bar{l}-1} c_{n}+\left(1-w_{\bar{l}-1}\right) \bar{c}_{e}+\hat{k}\right)+c_{n}\left(w_{\bar{l}} c_{n}+\left(1-w_{\bar{l}}\right) \bar{c}_{e}+\hat{k}\right) \\
= & \left(\hat{k}+\bar{c}_{e}-c_{n}-c_{e} w_{\bar{l}}+c_{n} w_{\bar{l}}\right)\left(\hat{k}-\bar{c}_{e} w_{\bar{l}-1}+c_{n} w_{\bar{l}-1}+\bar{c}_{e} w_{\bar{l}}-c_{n} w_{\bar{l}}\right)+\left(W_{I I .1}-W_{I I .0}\right) \sigma_{e}^{2} \\
= & \left(\left(1-w_{\bar{l}}\right)\left(\bar{c}_{e}-c_{n}\right)+\hat{k}\right)\left(\left(w_{\bar{l}}-w_{\bar{l}-1}\right)\left(\bar{c}_{e}-c_{n}\right)+\hat{k}\right)+W_{I I}(N) \sigma_{e}^{2} \\
= & \left(w_{d}\left(\bar{c}_{e}-c_{n}\right)+\hat{k}\right)^{2}+W_{I I}(N) \sigma_{e}^{2}
\end{aligned}
$$

where $w_{d}=\left(1-w_{\bar{l}}\right)=\left(w_{\bar{l}}-w_{\bar{l}-1}\right)$ and

$W_{I I}(N)=\left(W_{I I .1}(N)-W_{I I .0}(N)\right)=\sum_{i=\mathbb{L}_{0}} w_{\bar{l}-|i|}\left(w_{\bar{l}-|i+1|}-w_{\bar{l}-|i|}\right)>0$.

To compute $\bar{W}_{I I}$, note that for $N$ large enough $W_{I I}(N) \simeq \sum_{i=\underline{l}}^{\bar{l}}\left(y z^{|i|} \cdot y z^{|i+1|}\right)-\sum_{i=\underline{l}}^{\bar{i}}\left(y x^{-|i|}\right)^{2}-$ $y\left(y x^{-1}-y\right)=y^{2} \frac{x^{-1}-1}{x^{-1}+1}-y^{2}\left(x^{-1}-1\right)=\frac{y^{2}}{x} \frac{1-x^{-1}}{1+x^{-1}}=\frac{2}{9} \sqrt{3}-\frac{1}{3}=0.051567$.

b) For (27) to be decreasing in $c_{n}$, it sufficient that $\left(w_{d}\left(\bar{c}_{e}-c_{n}\right)+\hat{k}\right) \geq 0$. First, for every $G_{e}$, $\left|\bar{c}_{e}-c_{n}\right| \leq\left|c_{H}-c_{L}\right|$. Hence $\left(w_{d}\left(\bar{c}_{e}-c_{n}\right)+\hat{k}\right) \geq 0$ holds if $\left|c_{H}-c_{L}\right|<\frac{1}{w_{d}} \hat{k}$, which is satisfied by (12).

Proof of Lemma 3. Omitted.

Proof of Lemma 4. First inequality. By totally differentiating (30) with respect to $\alpha^{*}$ and $M$, we obtain: $\frac{d \alpha^{*}}{d M}=-\Phi_{M} / \Phi_{\alpha}$ which is negative since $\Phi_{M}<0$ from Lemma 3, and $\Phi_{\alpha}<0$ from Assumption 2. The second and the third inequalities are derived using similar arguments.

Proof of Lemma 5. Note that $\frac{d}{d M} E_{G_{\alpha^{*}(M)}}\left[\tilde{\Pi}_{n} \mid, M\right]=\frac{d \alpha^{*}(M)}{d M} \cdot \Phi\left[\alpha^{*}(M), M\right] G_{\alpha}\left(\alpha^{*}(M)\right)+$ $\int_{c_{L}}^{\alpha^{*}(M)} \frac{d}{d M}\left(\phi\left(\alpha^{*}(M), c_{n}, M, \sigma\right)\right) d G\left(c_{n}\right)$. Since the first addendum is null due to the zero-profit condition (30), a necessary and sufficient condition for monotonicity is that $\int_{c_{L}}^{\alpha} \frac{d}{d M} \phi\left(\alpha, c_{n}, M, \sigma\right)<$ 0 . However a sufficient condition is:

$$
\frac{d}{d M} \phi\left(\alpha^{*}(M), c_{n}, M, \sigma\right)=\phi_{\alpha}\left(\alpha^{*}(M), c_{n}, M, \sigma\right) \frac{d \alpha^{*}}{d M}+\phi_{M}\left(\alpha^{*}(M), c_{n}, M, \sigma\right)<0
$$

for any $c_{n} \leq \alpha$. Because $\phi_{M}<0$ from Lemma 3 and $\phi_{\alpha}<0$ from Assumption 2.(B), then $\phi\left(c_{H}, c_{H}, M, \sigma\right)>0$ implies $\frac{d \alpha^{*}}{d M}=0$, and (41) is satisfied. When $\phi\left(c_{H}, c_{H}, M, \sigma\right)<0, \frac{d \alpha^{*}}{d M}=$ $-\frac{\phi_{M}}{\phi_{\alpha}+\phi_{c_{n}}}\left(\alpha^{*}(M), \alpha^{*}(M), M, \sigma\right)$. Plugging the previous equation in (41) and dividing by 
$-\phi_{\alpha}\left(\alpha^{*}(M), c_{n}, M, \sigma\right)$, we obtain:

$$
\frac{\phi_{M}\left(\alpha^{*}(M), \alpha^{*}(M), M, \sigma\right)}{\phi_{\alpha}\left(\alpha^{*}(M), \alpha^{*}(M), M, \sigma\right)+\phi_{c_{n}}\left(\alpha^{*}(M), \alpha^{*}(M), M, \sigma\right)}<\frac{\phi_{M}\left(\alpha^{*}(M), c_{n}, M, \sigma\right)}{\phi_{\alpha}\left(\alpha^{*}(M), c_{n}, M, \sigma\right)}
$$

which holds thanks to Assumption 2.(C).

Proof of Proposition 8. The second and third inequalities are obtained by totally differentiating (33). The first inequality comes from the observation that dividing both sides of (34) the left-hand side is independent of $D$.

Proof of Proposition 9. First inequality - Totally differentiating (33), and recalling that from Lemma $5: \frac{d}{d M} E_{G_{\alpha^{*}(M)}^{\sigma}}\left[\tilde{\Pi}_{n} \mid, M\right]<0$, it follows that:

$\operatorname{sign}\left[\frac{d M_{S}}{d \sigma}\right]=\operatorname{sign}\left[\frac{d}{d \sigma} E_{G_{\alpha^{*}(M)}^{\sigma}}\left[\tilde{\Pi}_{n} \mid, M\right]\right]$. Note that:

$\frac{d}{d \sigma} \int_{c_{L}}^{\alpha^{*}}\left(\phi\left(\alpha^{*}, c_{n}, M, \sigma\right)\right) g\left(c_{n}\right) d c_{n}=$ $\frac{d \alpha^{*}}{d \sigma} \cdot \phi\left(\alpha^{*}, \alpha^{*}, M, \sigma\right) g^{\sigma}\left(\alpha^{*}\right)-\frac{d c_{L}}{d \sigma} \cdot \phi\left(\alpha^{*}, c_{L}, M, \sigma\right) g^{\sigma}\left(c_{L}\right)+\int_{c_{L}}^{\alpha^{*}} \frac{d}{d \sigma}\left(\phi\left(\alpha^{*}, c_{n}, M, \sigma\right) g^{\sigma}\left(c_{n}\right)\right) d c_{n}$,

where $\phi\left(\alpha^{*}, \alpha^{*}, M, \sigma\right)=0$. After expanding the last term:

$$
\begin{aligned}
& -\frac{d c_{L}}{d \sigma} \phi\left(\alpha^{*}, c_{L}, M, \sigma\right) g^{\sigma}\left(c_{L}\right)+\int_{c_{L}}^{\alpha^{*}} \phi\left(\alpha^{*}, c_{n}, M, \sigma\right) \frac{d g^{\sigma}\left(c_{n}\right)}{d \sigma} d c_{n}+ \\
& \int_{c_{L}}^{\alpha^{*}}\left(\frac{d \alpha^{*}}{d \sigma} \phi_{\alpha}\left(\alpha^{*}, c_{n}, M, \sigma\right)+\phi_{\sigma}\left(\alpha^{*}, c_{n}, M, \sigma\right)\right) g^{\sigma}\left(c_{n}\right) d c_{n}
\end{aligned}
$$

The last term is positive due to Assumption 2.(C) (see also the proof of Lemma 5), therefore a sufficient condition for $\frac{d M_{S}}{d \sigma}>0$ is that the first two terms are positive. Because $\frac{d c_{L}}{d \sigma}=\frac{1}{\sigma}\left(c_{L}-\bar{c}\right)<0$ and $\frac{d}{d \sigma} g^{\sigma}\left(c_{n}\right)=-\frac{1}{\sigma}\left(g^{\sigma}\left(c_{n}\right)+\left(c_{n}-\bar{c}\right) d g^{\sigma}\left(c_{n}\right) / d c_{n}\right)$, and remembering that $\phi\left(\alpha^{*}, \alpha^{*}, M, \sigma\right)=0$, then under Assumption 2.(D) $\frac{d M_{S}}{d \sigma}>0$ is always satisfied.

(Second inequality) If $\Phi>0$, then all firms stay in the market: $\alpha^{*}=c_{H}$ and therefore $\frac{d \alpha^{*}}{d \sigma}>0$. Otherwise if $\Phi<0$, then $\frac{d \alpha^{*}}{d \sigma}=\frac{d \alpha^{*}}{d M} \frac{d M_{S}}{d \sigma}<0$, from Lemma 4 .

\section{References}

[1] Aiura, Hiroshi and Yasuhiro Sato. 2008. "Welfare properties of spatial competition with location-dependent costs". Regional Science and Urban Economics, 38(1): 3248. 
[2] Anderson, Simon P. and de Palma, André. 2000. "From Local to Global Competition". European Economic Review, 44(3): 423-448.

[3] Bartelsman, Eric J. and Mark Doms, 2000, "Understanding Productivity: Lessons from Longitudinal Microdata ”. Journal of Economic Literature, 38(3): 569-594.

[4] Baskar, V. and Ted To. 2004. "Is perfect price discrimination really efficient? An analysis of free entry equilibria". RAND Journal of Economics, 35: 762-776.

[5] Bester, Helmut. 1992. "Bertrand Equilibrium in a Differentiated Duopoly". International Economic Review, 33(2): 433-448.

[6] Bhatia Rajendra, 1997. "Matrix Analysis". Springer-Verlag: New York.

[7] Bouckaert, Jan. 2000. "Monopolistic competition with a mail order business". Economics Letters, 66: 303-310.

[8] Chamberlin, Edward, H. 1956. "The Theory of Monopolistic Competition". Harvard University Press: Cambridge.

[9] Chen,Yongmin and Riordan, Michael H. 2007. "Price and Variety in the Spokes Model". Economic Journal, 117(522): 897-921.

[10] Creane, Anthony. 2007. "Note on uncertainty and socially excessive entry". International Journal of Economic Theory 3(4): 329-334.

[11] d'Aspremont Claude, J.J. Gabszewicz, and J.-F. Thisse, 1979, "On Hotelling's "Stability in Competition". Econometrica, 47(5): 1145-1150.

[12] Dasgupta Partha and Eric Maskin, 1986. "The Existence of Equilibrium in Discontinuous Economic Games, II: Applications". Review of Economic Studies, 53(1):27-41

[13] Dunne, T., Roberts, M.J. and Samuelson, L., 1989. "The Growth and Failure of U.S. Manufacturing Plants". Quarterly Journal of Economics, 104:671-698.

[14] Eaton, Curtis B. and Lipsey, Richard G., 1978. "Freedom of Entry and the Existence of Pure Profit". Economic Journal, 88(351): 455-69.

[15] Foster, L., Haltiwanger, J. and Krizan, C.J., 2006. "Market Selection, Reallocation and Restructuring in the U.S. Retail Trade Sector in the 1990s". Review of Economics and Statistics, 88:748-758. 
[16] Levin, Dan and Peck, James, 2003. "To Grab for the Market or to Bide One's Time: A Dynamic Model of Entry". Rand Journal of Economics, 34(3): 536-556.

[17] Liu, Qihong and Serfes, Constantinos, 2005. "Inperfect price discrimination, market structure, and efficiency". Canadian Journal of Economics, 38(4): 1191-1203.

[18] Melitz, M. and Ottaviano, Gianmarco, 2008, "Market Size, Trade, and Productivity". The Review of Economic Studies, 75(1):295-316.

[19] Montagna, Catia, 1995, "Monopolistic Competition with Firm-Specific Costs". Oxford Economic Papers, 47: 318-328.

[20] Nocke, Volker 2006. "A Gap For Me: Entrepreneurs and Entry". Journal of the European Economic Association, 4(5):929-956.

[21] Piga, Claudio, A. 1998. "A Dynamic Model of Advertising and Product Differentiation". Review of Industrial Organization, 13(5): 509-522.

[22] Pinkse, Joris, Margaret, E., Slade and Craig Brett. 2002. "Spatial Price Competition: a Semiparametric Approach." Econometrica, 70(3): 1111-1153.

[23] Rothschild, Robert. 1982. "Competitive Behaviour in Chain-Linked Markets." Journal of Industrial Economics, 33(1-2): 57-67.

[24] Salop, Steven C. 1979. "Monopolistic Competition with Outside Goods." Bell Journal of Economics, 10(1): 141-156.

[25] Schaumans, Catherine and Frank Verboven. 2006. "Entry and Regulation - Evidence from the Health Care Professions.", CEPR Discussion Paper No. 5482, February, forthcoming Rand Journal of Economics.

[26] Spulber, Daniel F. 1995. "Bertrand Competition when Rivals' Costs are Unknown". Journal of Industrial Economics, XLIII(1):1-11.

[27] Syverson, Chad 2007. "Prices, Spatial Competition and Heterogeneous Producers: An Empirical Test". Journal of Industrial Economics, LV(2):197-222.

[28] Syverson, Chad 2004. "Market Structure and Productivity: A Concrete Example". Journal of Political Economy, 112(6):1181-1222.

[29] Syverson, Chad 2001. "Market Structure and Productivity: A Concrete Example" U.S. Bureau of the Census, Working Paper, April. 
[30] Tirole, Jean. 1988. "The Theory of Industrial Organization". MIT Press: Cambridge, Mass., USA.

[31] Vogel, Jonathan. 2008. "Spatial Competition with Heterogeneous Firms". Journal of Political Economy,116(3):423-466.

[32] Waterson, Michael, 1990, "Product Differentiation and Profitability: An Asymmetric Model", Journal of Industrial Economics, 39: 113-130.

[33] von Ungern-Sternberg, Thomas. 1991. "Monopolistic Competition on the Pyramid." Journal of Industrial Economics, 39(4): 355-368. 


\section{$7 \quad$ Tables and Figures}

Table 1: The sequence of equilibrium prices' weights (percentage values).

\begin{tabular}{|c|c|c|c|c|c|c|c|c|c|c|c|}
\hline$N$ & $\underline{l}$ & $\bar{l}$ & $w_{\bar{l}}$ & $w_{\bar{l}-1}$ & $w_{\bar{l}-2}$ & $w_{\bar{l}-3}$ & $w_{\bar{l}-4}$ & $w_{\bar{l}-5}$ & $w_{\bar{l}-6}$ & $w_{\bar{l}-7}$ & $R$ \\
\hline 2 & 0 & 1 & 66.667 & 33.333 & & & & & & & $\overline{0.000}$ \\
\hline 3 & -1 & 1 & 60.000 & 20.000 & & & & & & & 0.000 \\
\hline 4 & -1 & 2 & 58.333 & 16.667 & 8.333 & & & & & & 0.000 \\
\hline 5 & -2 & 2 & 57.895 & 15.789 & 5.263 & & & & & & 0.000 \\
\hline 6 & -2 & 3 & 57.778 & 15.556 & 4.444 & 2.222 & & & & & 0.000 \\
\hline 7 & -3 & 3 & 57.747 & 15.493 & 4.225 & 1.408 & & & & & 0.000 \\
\hline 8 & -3 & 4 & 57.738 & 15.476 & 4.167 & 1.190 & 0.595 & & & & 0.595 \\
\hline 9 & -4 & 4 & 57.736 & 15.471 & 4.151 & 1.132 & 0.377 & & & & 0.755 \\
\hline 10 & -4 & 5 & 57.735 & 15.470 & 4.147 & 1.116 & 0.319 & 0.159 & & & 0.798 \\
\hline 11 & -5 & 5 & 57.735 & 15.470 & 4.146 & 1.112 & 0.304 & 0.101 & & & 0.809 \\
\hline 12 & -5 & 6 & 57.735 & 15.470 & 4.145 & 1.111 & 0.299 & 0.085 & 0.043 & & 0.814 \\
\hline 13 & -6 & 6 & 57.735 & 15.470 & 4.145 & 1.111 & 0.298 & 0.081 & 0.027 & & 0.813 \\
\hline 14 & -6 & 7 & 57.735 & 15.470 & 4.145 & 1.111 & 0.298 & 0.080 & 0.023 & 0.011 & 0.813 \\
\hline 15 & -7 & 7 & 57.735 & 15.470 & 4.145 & 1.111 & 0.298 & 0.080 & 0.022 & 0.007 & 0.813 \\
\hline 20 & -9 & 10 & 57.735 & 15.470 & 4.145 & 1.111 & 0.298 & 0.080 & 0.021 & 0.006 & 0.813 \\
\hline$\infty$ & $-\infty$ & $\infty$ & 57.735 & 15.470 & 4.145 & 1.111 & 0.298 & 0.080 & 0.021 & 0.006 & 0.813 \\
\hline
\end{tabular}


Table 2: The sequence of equilibrium parameters in the Entry Games (percentage values).

\begin{tabular}{ccccc}
\hline$N$ & $\rho_{c}$ & $\rho$ & $W_{I}$ & $W_{I I}$ \\
\hline \hline 2 & 300.00 & 300.000 & 22.222 & 11.111 \\
\hline 3 & 250.00 & 69.987 & 24.000 & 8.000 \\
\hline 4 & 200.00 & 62.436 & 23.611 & 6.250 \\
\hline 5 & 190.00 & 61.356 & 23.269 & 5.540 \\
\hline 6 & 180.00 & 61.076 & 23.111 & 5.284 \\
\hline 7 & 177.50 & 61.001 & 23.051 & 5.197 \\
\hline 8 & 175.00 & 60.881 & 23.030 & 5.169 \\
\hline 9 & 174.34 & 60.976 & 23.023 & 5.161 \\
\hline 10 & 173.68 & 60.974 & 23.021 & 5.158 \\
\hline 11 & 173.51 & 60.974 & 23.020 & 5.157 \\
\hline 12 & 173.33 & 60.974 & 23.020 & 5.157 \\
\hline 13 & 173.29 & 60.974 & 23.020 & 5.157 \\
\hline 14 & 173.24 & 60.974 & 23.020 & 5.157 \\
\hline 15 & 173.23 & 60.974 & 23.020 & 5.157 \\
\hline 20 & 173.21 & 60.974 & 23.020 & 5.157 \\
\hline$\infty$ & 173.21 & 60.974 & 23.020 & 5.157 \\
\hline
\end{tabular}




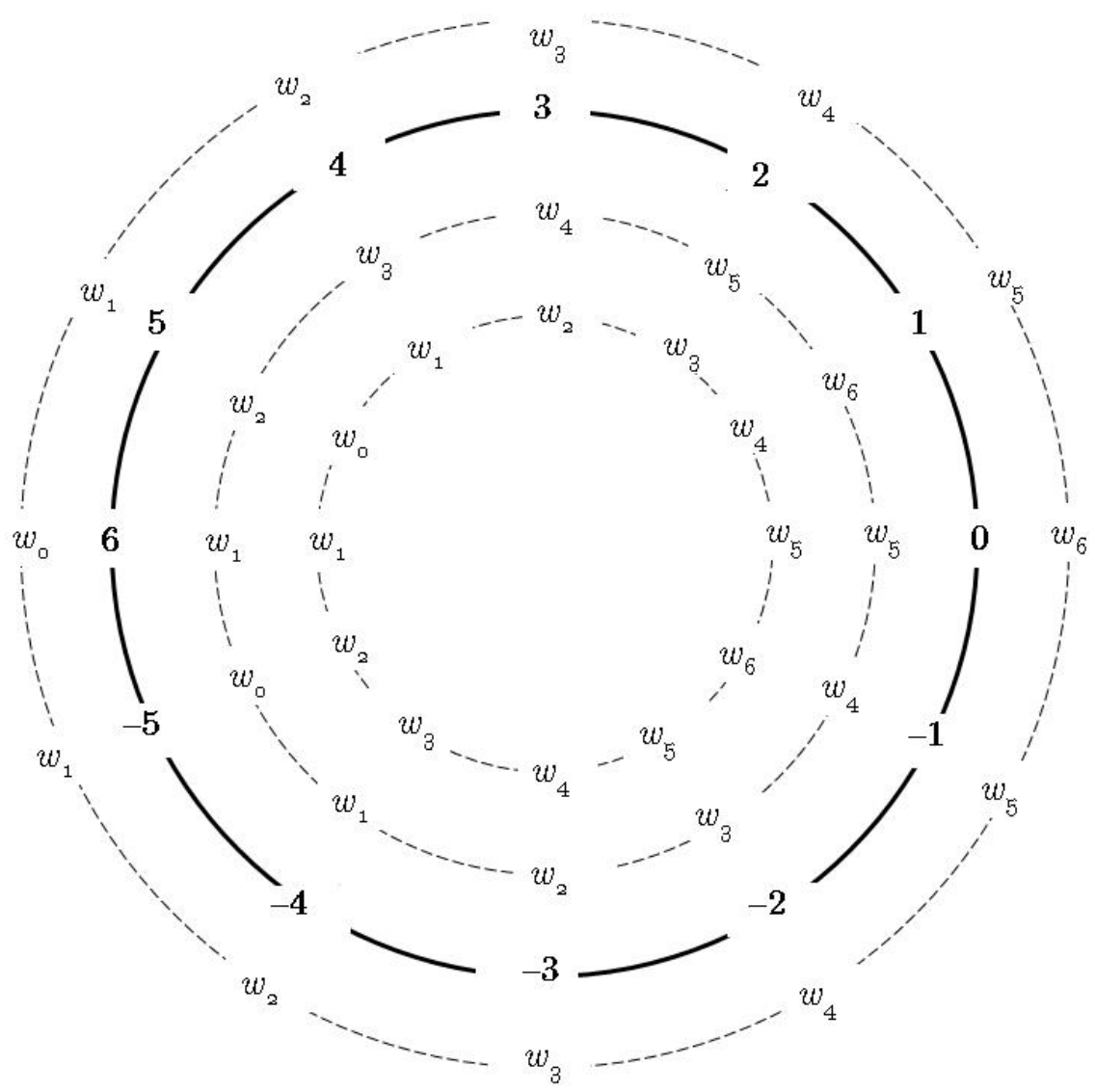

Figure 1: The bold line describes the location of the firms. The external dashed circumference denotes the weights given by firm 0 to the costs of the firms located at any other point. The internal first (second) dashed circumference denotes the weights given by firm $+1(-1)$ to the costs of any other firm. 


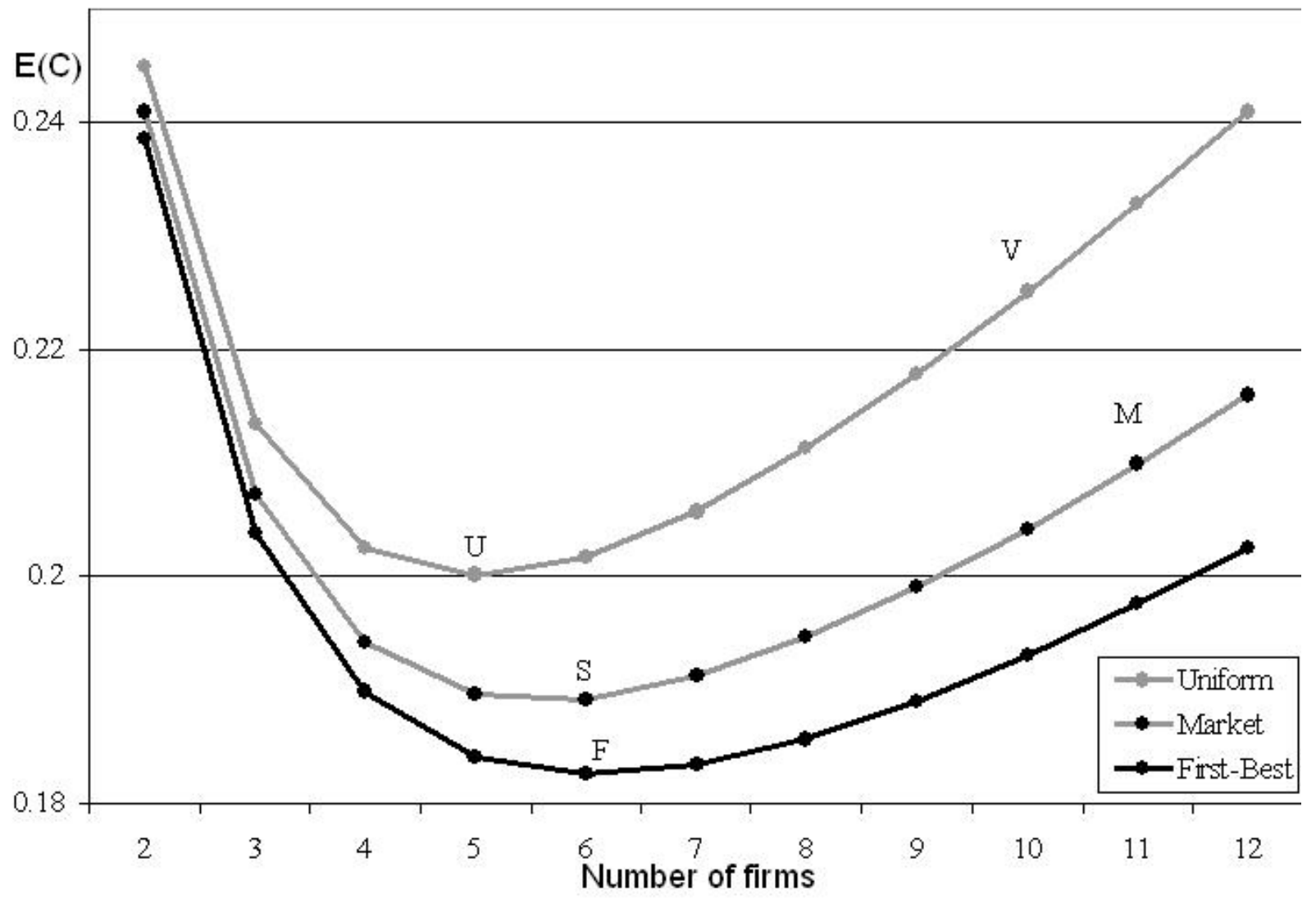

Figure 2: Expected average total cost. $F$ is the social optimum; $M$ is the free-market outcome; $S$ is the second best solution (the social planner's choice of entrants when they set market prices). Under uniform pricing, the planner's choice is in $U$ ( $V$ is the market outcome with homogeneous costs). 


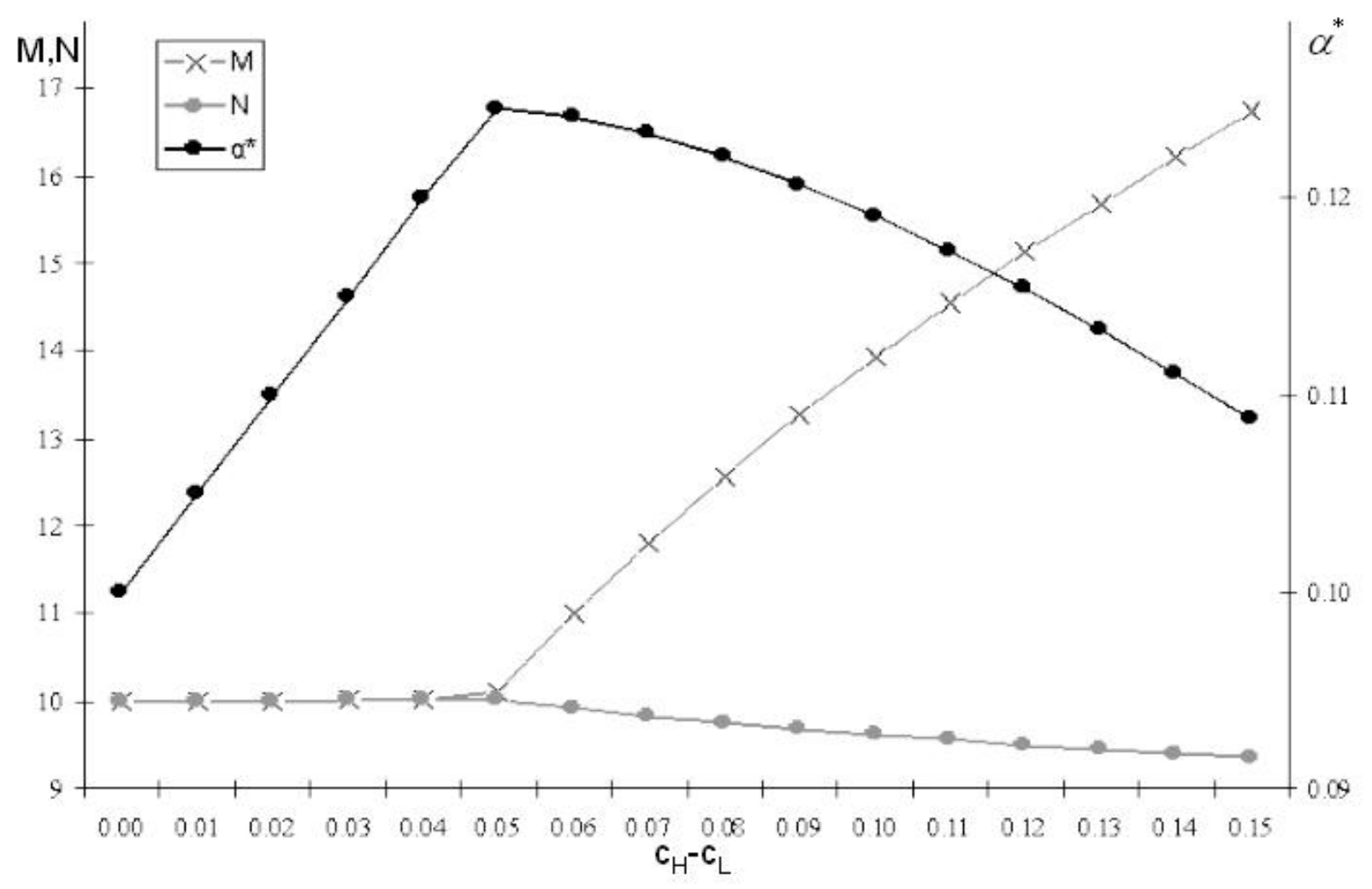

Figure 3: The optimal number firms in the pre-entry stage $M$ and in the production stage $N$ (left scale). Optimal entry threshold (right scale). 


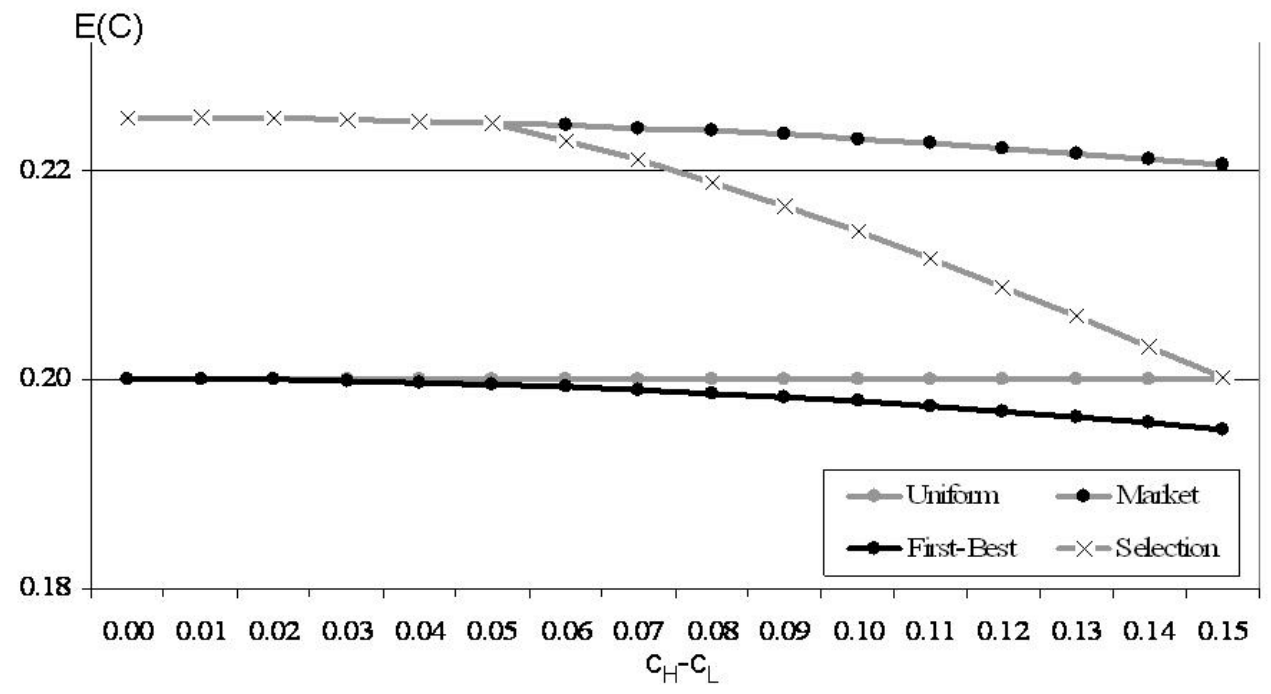

Figure 4: Expected average costs with regulation (uniform and first-best pricing) and without (free market and market with selection). 